\title{
Competitive balance: Information disclosure and discrimination in an asymmetric contest
}

\author{
Derek J. Clark ${ }^{\mathrm{a}}$, Tapas Kundu ${ }^{\mathrm{b}, *}$ \\ a School of Business and Economics, UiT the Arctic University of Norway, Norway \\ ${ }^{\mathrm{b}}$ Oslo Business School, Oslo Metropolitan University, Norway
}

\section{A R T I C L E I N F O}

\section{Article history:}

Received 18 August 2020

Revised 21 December 2020

Accepted 30 January 2021

\section{JEL classification:}

D02

D72

D82

Keywords:

Asymmetric contest

Information design

Discrimination

\begin{abstract}
A B S T R A C T
We study a design problem for an effort-maximizing principal in a two-player contest with two dimensions of asymmetry. Players have different skill levels and an information gap exists, as only one player knows the skill difference. The principal has two policy instruments to redress the lack of competitive balance due to asymmetry; she can commit to an information-disclosing mechanism, and she can discriminate one of the players by biasing his effort. We characterize the optimal level of discrimination to maximize aggregate effort, showing how this inextricably determines the choice of information disclosure. Applications are found in newcomer-incumbent situations in an internal labor market, sales-force management, and research contests.
\end{abstract}

(c) 2021 The Author(s). Published by Elsevier B.V. This is an open access article under the CC BY license (http://creativecommons.org/licenses/by/4.0/)

\section{Introduction}

Competition in social, political and economic spheres is often analyzed as a contest in which resources are sunk in order to win a prize. Numerous applications of these frameworks can be found in the literature relating to conflict and warfare, lobbying, elections, internal and external labor markets or various types of research competition. ${ }^{1}$ A common theme in much of the existing work is how the characteristics of the competitors and structure of the contest affect the amount of resources or effort used in the competition, and how a contest designer may attempt to influence this; the most usual assumption is that the designer wishes to maximize the resources expended. ${ }^{2}$ We consider a contest between an incumbent and a newcomer for a fixed prize. Such situations are often characterized by the newcomer having better information than both the rival and the contest designer about attributes such as own ability that are relevant to the playing of the contest. The difference in this attribute may be large or small, and it is not certain that the incumbent is the superior contestant. Designing the contest to maximize effort in this situation is not a trivial exercise for the principal since it involves negotiating two dimensions of heterogeneity. First, hidden information makes the return to effort uncertain for the uninformed

\footnotetext{
We would like to thank two anonymous referees for insightful comments. Any remaining errors are our own.

* Corresponding author.

E-mail addresses: derek.clark@uit.no (D.J. Clark), tapas.kundu@oslomet.no (T. Kundu).

1 See Konrad et al. (2009) and the references therein.

${ }^{2}$ Other aims are possible. In some contexts achieving a close contest may be the objective (Runkel, 2006), or maximizing participation (Azmat and Möller, 2009), or securing the highest quality winner (Serena, 2017b).
} 
player, discouraging effort. ${ }^{3}$ Second, heterogeneity, as captured by a relative skill disparity, is generally acknowledged to be one factor that limits resource use in contest settings (see Chowdhury et al., 2020). A player with a large relative ability, or a large relative valuation of winning, may intimidate opponents into submitting low efforts, and can hence reduce his own efforts and still win with a large probability. ${ }^{4}$

In this paper, we set up a simple model that effectively captures the incumbent-newcomer scenario, and in which the principal has two policy instruments at her disposal. She can commit to a signaling mechanism which may reveal - at least partially - the hidden information; furthermore, she can use a policy which treats one of the players preferentially by biasing positively his effort level in the contest. ${ }^{5}$ We demonstrate that there is an interesting interplay between these two policy instruments, and that the optimal level and direction of discrimination inextricably determines the choice of information disclosure. Furthermore, we show that the principal must be mindful of the fact that her choices may affect the incentives of the less skilled to exert effort in the contest. ${ }^{6}$

Our contribution is twofold. First, we analyze a model in which there is asymmetric information about the abilities of the contestants and show how the skill differential and the existing discrimination policy affects the incentives of the principal to reveal information; second we allow the principal to choose the optimal level of discrimination, in which she has to take account of how this affects the optimal disclosure of information. Our model features uncertainty about the characteristics of the contestants, rather than the value of the contested prize. Specifically, there is a skill differential between the two players in carrying out the contest task, the relative value of which is known only to one player, the newcomer; the skill differential can be large or small, and may positively favor either player. Our modeling assumption is that a player's skill is multiplied by his effort to give his effective level of contest effort which directly affects the success probabilities. The uninformed incumbent and principal have a prior distribution over two possible values of the relative skill. In seeking to maximize the expected effort from the contest, the designer can commit to a set of signals that are sent after the state is determined. Furthermore, we introduce a discrimination parameter which changes the relative productivity of the contestants' effort in determining the probability of success. The discrimination parameter affects effective contest effort multiplicatively, scaling a contestant's effort up or down depending on the direction of the discrimination; given that skill also affects effective contest effort multiplicatively, we show that a multiplicative discrimination parameter is a better policy instrument for the principal than for example an additive one.

When the discrimination parameter is fixed, the designer can influence effort only through the signaling mechanism. We calculate the optimal signals set by the principal, depending on the fixed discrimination policy. Furthermore, we subsequently allow her to choose this parameter in order to achieve the maximal amount of effort possible. In so doing, she must be mindful of the fact that the choice of the magnitude and direction of discrimination affects the optimal policy of information disclosure. We show that the designer will not want to implement a level of discrimination that involves partial disclosure of the hidden information. She chooses optimally between values of the discrimination parameter for which no disclosure or full disclosure is optimal, and we show how this is connected to the prior beliefs of the uninformed incumbent. When it is thought that the informed newcomer is very likely to be skill-inferior then the designer does not benefit from revealing this to the uninformed opponent, and she chooses to discriminate in favor of the informed (but likely low-skilled) player; we show further how the magnitude of the discrimination depends on the skill level. On the other hand, when the uninformed incumbent thinks that it is likely that the opponent will be highly skilled, the designer must alleviate riskiness of effort for this player by revealing the true state; she will also discriminate against the informed player to encourage effort by both.

One can imagine several applications in which a newcomer competes against an incumbent for a prize, and the ability of the incumbent is known by all, but the newcomer has hidden talent. ${ }^{7}$ An internal labor market has features in common with our framework in which an insider and an outsider to a firm compete for a position or a promotion. The outsider knows his own skill level, but the insider is uncertain of the quality of the rival. In the context of a tournament model, Chan (1996) analyses the preferential treatment of one type of candidate depending on the unknown skill level of external workers. If they are expected to be highly skilled, the performance of the firm can be improved by giving preferential treatment to internal candidates; if external candidates are of little threat in terms of skill level, then they can be given an advantage in the promotion contest to incentivize the internal candidates. This is a simple mechanism for leveling the playing field. Our model develops this approach by making manipulation of the information structure a policy instrument in the contest design. In this case, the signaling mechanism that we consider can be likened to the use of aptitude tests

\footnotetext{
3 Asymmetry in what the players know about the structure of the contest can generally lead to a low effort level (Wärneryd, 2003).

${ }^{4}$ In a dynamic race setting, Konrad and Kovenock (2009) investigate the discouragement effect that arises as one competitor nears the finishing line, causing opponents to simply give up.

${ }^{5}$ In this way we combine incentive manipulation with information provision, which Kamenica (2019, footnote 2) regards as a promising area for future research.

${ }^{6}$ Here, the principal chooses whether and how to disclose information. Denter et al. (2020) consider a situation where the agents themselves can choose to reveal information. See also Epstein and Mealem (2013).

7 Denter et al. (2020) also consider this framework, analyzing the information disclosure decisions made by the newcomer himself by choosing to show off his talent, or lie low.
} 
by firms to glean some information about their skill level. ${ }^{8}$ The informativeness of the signal can be chosen by the firm according to the test design. A fully informative signal about an agent's ability could reveal many dimensions of skill through tests of cognitive ability, problem-solving, critical thinking and decision making. A less informative signal may only consider one of these dimensions. In sales-force management, an established seller may face competition from an outside challenger and the firm may choose to disclose information about past sales performance of the challenger; this information may be coarse (number of sales or revenue achieved) or finer (giving details of sales territories, product information, and a relative comparison with peers). Discrimination in this case can be thought of as less administrative duties, better access to backoffice resources, more training, and better territories; see, e.g., Skiera and Albers (1998), Farrell and Hakstian (2001), and Krishnamoorthy et al. (2005).

Research contests between competing teams also fit our framework. The effort made by each applicant can be thought of as building up the quality of the team that is competing for a research grant; one team may already be well established whereas a challenger is up-and-coming and not tested. In this case, the research sponsor could grant the new team a preproject to gather information about its relative skill level. ${ }^{9}$ Discrimination in this case could involve preferential treatment of young researchers or aid in writing a good proposal. Many large corporations run internal innovation competitions which see employees (or teams of employees) compete with each other in order to achieve further funding for their projects. ${ }^{10}$ Rathi (2014) documents that Thompson Reuters, the US Department of Health and Human Services, Reed Elsevier and TC Transcontinental (the largest printing company in Canada) use different forms of innovation contest, releasing information about previous contests and findings, and using internal mentors as a way of giving an advantage in the competition. Similarly, one may think of procurement contracting as fitting our model, in which an incumbent entrepreneur is well known, whereas a challenger has hidden qualities. Instructing the newcomer to develop a prototype, and revealing the results of its testing, is a way in which information can potentially be garnered and released. Further mechanisms of information disclosure may be legally specified. Zhang and Zhou (2016) note that political candidates in the US are required by the Federal Election Campaign Act to reveal campaign contributions and expenditures; this provides information about the financial support base of the candidates.

\section{Related Literature}

We draw together three strands of literature in this paper. One relates to discrimination in contests, the second to asymmetric information structures in contests, and the third to the use of signaling mechanisms to reveal hidden information, otherwise known as Bayesian persuasion (Kamenica and Gentzkow, 2011). ${ }^{11}$ Chowdhury et al. (2020) and Mealem and Nitzan (2016) discuss different forms of discrimination aimed at leveling the playing field to achieve competitive balance in asymmetric contests. Instruments at the disposal of an effort-maximizing principal include exclusion of strong players (Baye et al., 1993), caps on efforts (Che and Gale, 1997) or various forms of discrimination such as differential taxation of the prize, or giving head starts or handicaps to some players (see the survey by Mealem and Nitzan, 2016). In the latter case, one may affect the structure of the contest environment through the probability of success by giving a head start or biasing the efforts of one or more players. For the two-player case, Franke (2012) shows how biasing the efforts optimally in relation to competitors' cost of effort (or equivalently prize valuation) leads to maximal contest effort. ${ }^{12}$ Fu and Wu (2020) show that multiplicative biases outperform additive heads starts in an n-player lottery contest with heterogeneous contestants differing in their prize valuations. The principal pursues a broad range of objectives, including total effort maximization.

Leaving the framework of complete information, Hurley and Shogren (1998a), Hurley and Shogren (1998b) and Wärneryd (2003) consider how asymmetries in information can affect contest behavior. Particularly relevant to our work is their focus on cases with one-sided informational asymmetry, where one player has better information than the competitor. ${ }^{13}$ Suppose that in a two-player framework, one knows the exact value of the prize but the other knows only the underlying distribution. The effort of the uninformed player is then likened by Hurley and Shogren (1998a) to a risky input which tends to decrease effort in equilibrium, a finding that is reinforced by Wärneryd (2003) who shows that the twoplayer lottery contest with asymmetric information yields lower equilibrium effort than when the players are symmetrically informed or uninformed.

Our paper is closest to that of Epstein and Mealem (2013) who attempt to bridge the gap between one-sided incomplete information on the one hand, and information disclosure on the other. We use their basic contest framework, and extend it in two important directions. First, we allow for exogenous and endogenous discrimination of the contestants, showing

\footnotetext{
8 Several large firms use aptitude tests in hiring such as Apple, Samsung, Microsoft and Nike. See https://www.aptitude-test.com/blog/articles/ 10-major-companies-that-use-aptitude-testing/. SHL's Global Assessment Trends Report in 2018 documents that $76 \%$ of organizations with over 100 employees use aptitude and personality tests in hiring decisions. See https://www.shl.com/en/assessments/trends/.

9 Serena (2017a) mentions several research contests in which there is an initial stage in which information on the rivals may be gathered and revealed, before final proposals are made. These include the Horizon 2020 submissions to the European Research Council and design competitions run by the Royal Australian Institute of Architects.

10 See Adamczyk et al. (2012) for a review of research on innovation contests, and Höber (2017) for internal contests.

11 Our focus is on a lottery contest, originating in Tullock (1980), rather than an all-pay auction (Hillman and Riley, 1989). See Lu et al. (2018) for an analysis of information disclosure in an all-pay auction.

12 See also Epstein et al. (2013).

13 Serena (2017a) in contrast considers a model of information disclosure in a lottery contest with two-sided private information.
} 
how this affects contest incentives; second, we consider optimal information disclosure by an effort-maximizing principal. Epstein and Mealem (2013) consider the incentives of the informed player to reveal own type before it is known, in order to increase his own expected payoff from the contest. Whilst they consider information disclosure as a binary decision, our analysis covers a much wider specification of possibilities in which the principal can reveal all or no information at two extremes, but can also choose a set of signals to impart a particular posterior belief to the uninformed type when this is optimal. Furthermore, we analyze the interplay between this information disclosure and the optimal discrimination policy.

Manipulation of the information structure is an instrument that can be employed to achieve a specific goal. Kamenica and Gentzkow (2011) have operationalized a method of Bayesian persuasion in which a principal can commit to a stateconditional distribution of signals before realization of the state; this has been applied to a lottery contest by Zhang and Zhou (2016), Feng and Lu (2016) and Fu et al. (2016). The latter papers consider information disclosure about an unknown number of competitors, whilst Zhang and Zhou (2016) is more relevant for our analysis since it is a two-player contest. The asymmetric information relates to the value of the prize, and the effort-maximizing designer must reveal the state optimally by committing to a signaling mechanism. ${ }^{14}$ Kamenica and Gentzkow (2011) show generally that full disclosure is an optimal policy if the payoff of the sender (principal) as a function of the belief of the receiver (uninformed contestant) is globally convex, whilst no disclosure is best when it is globally concave; if the payoff function of the sender has concave and convex portions, then partial disclosure is optimal. Zhang and Zhou (2016) consider first a structure in which the hidden prize value is binary, which yields an expected effort function that is globally convex or concave depending on the valuation by the informed player and the two possible valuations of the uninformed; hence, a signal is optimal that gives either full disclosure of the hidden state, or no disclosure. Only when there are more than two possible valuations can partial disclosure appear, in which the signal reveals the true value of the prize imperfectly to the uninformed player. Our findings show that even for a binary distribution of the skill differential, partial disclosure can be optimal for some given level of discrimination, although the principal will not implement such a discrimination level if she can choose it. ${ }^{15}$

The paper is organized as follows. Section 2 sets up the basic contest and framework for information disclosure, and Section 3 solves for equilibrium effort levels under different informational assumptions. Section 4 considers the optimal information disclosure policy for a given level of discrimination, and the optimal direction and magnitude of discrimination is calculated in Section 5. We discuss our main modeling assumptions in Section 6, and Section 7 concludes. The Appendix contains proofs of our results. ${ }^{16}$

\section{Model}

Two risk-neutral agents, $N$ (newcomer) and $I$ (incumbent), compete for a fixed prize of value 1 in a contest designed by the principal $P$. The contest score achieved by player $I$ is simply given by his effort $e_{I}$, and the score of the newcomer is a multiple of his effort: $\alpha s e_{N}$, where $\alpha>0$ and $s>0$. The parameter $\alpha$ measures the degree of discrimination: $\alpha>1$ $(0<\alpha<1)$ implies that $N$ is positively (negatively) discriminated. This parameter is chosen optimally by the principal in the analysis below. The parameter $s$ is one of the primitives of the model, and measures $N$ 's relative skill: $s>1(0<s<1)$ implies that $N$ is superior (inferior) in skill. The success probabilities of $N$ and $I$, given an effort profile $\left(e_{N}, e_{I}\right), e_{N}, e_{I}>0$, are determined by a player's score relative to the total score in the contest:

$$
\rho_{N}=\frac{\alpha s e_{N}}{\alpha s e_{N}+e_{I}}, \quad \rho_{I}=\frac{e_{I}}{\alpha s e_{N}+e_{I}} .
$$

This contest success function is commonly used, and has been axiomatized by Clark and Riis (1998). ${ }^{17}$ Schaller and Skaperdas (2020) suggest the multiplicative approach taken here as a general way of capturing asymmetry in contests. The skill differential implies that the newcomer is a certain percentage better or inferior than the incumbent at carrying out the contest task, and it would then appear natural that the instrument of the principal should also be multiplicative. This is also in line with the approach to modeling discrimination taken by Epstein et al. (2011).18

We assume that the relative skill $s$ is the source of information asymmetry at the beginning of the game and is referred to as the state of the game. Player $N$ knows the state. Player $I$ and the principal do not know the state, but know that $N$ is fully informed. For simplicity, we assume that there are two possible states, one in which $N$ is superior and the other

\footnotetext{
14 Kamenica and Gentzkow (2011, p. 2599) discuss the plausibility of committing to a signaling mechanism, noting that: "Firms often commit to the information they will seek out in performance reviews. Academic departments follow fixed rules about the information they will solicit for midterm or tenure reviews".

15 A major difference between our model and Zhang and Zhou (2016) is the fact that our use of the discrimination parameter renders the relative asymmetry between the players a continuous variable, even though the skill differential takes one of two values. For some of these values partial disclosure is optimal. In Zhang and Zhou (2016), it is the relative valuation of the two players that captures the asymmetry, and this takes a finite number of values; either full disclosure or no disclosure is optimal for all of these values in their model.

16 Appendix B gives supplementary material relating to the analysis in Section 6.

17 If $\alpha s e_{A}+e_{B}=0$, we assume that the prize is not awarded. This does not occur in equilibrium, however.

18 "The bias is represented by the different values of a unit of effort made by the contestants", Epstein and Mealem (2013, p. 89). Modeling the skill differential as an additive head start is also a possibility. However, it is widely acknowledged that head starts tend to dampen contest effort in two-player contests (Franke et al., 2013). With several players they can, however, be a useful tool for encouraging the participation of desirable players (with a high prize valuation for example), and excluding others.
} 
in which $N$ is inferior. Specifically, $s$ can take only two values in $S:=\{x, y\}, y>1>x>0$, with prior probabilities $q \in(0,1)$ and $(1-q)$, respectively. This contest specification is the same as Epstein and Mealem (2013) who analyze the special case $\alpha=1$. In order to be able to isolate the effect of the discrimination policy on behavior, we assume that $y=\frac{1}{x}$ and $x \in(0,1)$; implications of relaxing this assumption are discussed in Section $6 .{ }^{19}$ Since $S=\left\{x, \frac{1}{x}\right\}$ contains only two values, a distribution over the state space can be expressed with a scaler $p \in[0,1]$ such that $p=\operatorname{Pr}[s=x]$. We will follow this convention, unless stated otherwise. In addition, we use the notation $p$ to denote a generic distribution wherever needed, while $q$ always refers to the prior, which is a parameter of the model.

\subsection{Information disclosure}

Before the state is realized, the principal commits to and publicly discloses a pair of state-conditional signal distributions $\left\{\pi(\cdot \mid x), \pi\left(\cdot \mid \frac{1}{x}\right)\right\}$ such that $\pi(\cdot \mid s) \in \Delta(M)$, where $M$ is a finite set of signals and $\Delta(M)$ is the set of all probability distributions over $M .^{20}$ Once the state $s$ is realized and revealed to $N$, nature draws a signal $m \in M$ from the distribution $\pi(\cdot \mid s)$. Both agents observe the signal and the uninformed agent $I$ updates his belief. We let $q_{m}$ denote $I$ 's posterior belief that $N$ is inferior, after observing a signal $m$, i.e., $q_{m}=\operatorname{Pr}[s=x \mid m]$. Note that since $N$ also observes the signal, $N$ can infer $q_{m}$.

The contest then takes places with agents exerting effort simultaneously. The cost of effort is linear and identical for each agent, with a constant marginal cost of one. The agent $i \in\{N, I\}$ chooses effort $e_{i} \geq 0$ to maximize his expected payoff, denoted by $v_{i}$. The principal's payoff is given by the total expected efforts and is denoted by $V_{P}$.

The timing of the game is as follows:

1. $P$ chooses the degree of discrimination $\alpha>0$, which is common knowledge;

2. $P$ commits to and publicly discloses a set of distributions $\left\{\pi(\cdot \mid x), \pi\left(\cdot \mid \frac{1}{x}\right)\right\}$ where $\pi(\cdot \mid s) \in \Delta(M)$, the set of all probability distributions over the signal space $M$;

3. Nature draws a realization of the state $s \in S$, which is revealed to player $N$. Next, nature draws a signal $m \in M$ from $\pi(\cdot \mid s)$. The signal $m$ is publicly observed, leading to a posterior belief distribution for $I$;

4. The contest takes place with $N$ and $I$ choosing effort simultaneously.

We study the perfect Bayesian equilibrium of the game.

\subsection{Discussion of the model assumptions}

We make certain assumptions in our model for analytical tractability. First, we consider only two possible states. When there are more than two states, the analysis of optimal information disclosure requires analyzing the convexity property of a function with a multi-dimensional domain. Since we characterize the information disclosure policy for all possible levels of discrimination, this analysis becomes quite intractable. In order to focus on the key issue of the optimal discrimination, we restrict the state space. Further, if both state values are above (below) one, then the principal can infer that the uninformed player $I$ is always inferior (superior), and the result on the direction of discrimination is straightforward. The more interesting case is the one in which the uninformed player can be either superior or inferior to the informed player. Our assumption that the two state values are reciprocal to each other allows us to analyze this interesting case in a straightforward manner. In Section 6, we show that the reduced model that we analyze is related to the general one by a simple isomorphic mapping. Our main results remain intact.

A final assumption worthy of comment is that the principal chooses the discrimination policy at the beginning of the game, so that it is not conditioned on any information that might be revealed about the state. Knowing the possible states of the world, the principal sets the policy in advance, regulating effort through the signaling mechanism; the principal only observes the state if the signal is fully revealing, and we show below that this is not always an optimal choice. The degree of discrimination and the commitment to the signal distributions can be chosen simultaneously without changing our results. The important point is that these are decided before any information is revealed about the state. Hence, the principal makes optimal decisions based upon the structure of the environment in which she operates, of which uncertainty over newcomer skill is an inherent feature. We think of the policy choice as being an impersonal and overarching principle that does not change according to the skill of the player that competes. This fits many competitive environments in which rules are made without knowing the exact set of participants, including research contests, sporting events, litigation, political competition, and internal labor markets.

\section{Contest}

We begin at stage 4 where the contest can take place under two possible information structures: (i) full information and (ii) asymmetric information.

\footnotetext{
19 See also the discussion of the main assumptions in Section 2.2.

${ }^{20}$ Kamenica (2019, p. 251) notes that "The key object in Bayesian persuasion models is the thing that Sender chooses, which goes by many names, including signal, signal structure, information structure, experiment, Blackwell experiment, or data-generating process." In line with Kamenica (2019), we refer to this as signal.
} 


\subsection{Full information}

Suppose both agents know the value of $s$. Agent $i$ chooses effort to maximize his expected payoff $v_{i}=\rho_{i}-e_{i}$. Lemma 1 documents the principal's expected payoff in the Nash equilibrium of the game. We use the notation $\mathbb{E}_{p}[f(s)]$ to denote the expected value of $f(s)$ given that $p=\operatorname{Pr}[s=x]$; therefore, $\mathbb{E}_{p}[f(s)]:=p f(x)+(1-p) f\left(\frac{1}{x}\right)$.

Lemma 1. The Nash equilibrium of the full information contest is symmetric, and the principal's ex ante expected payoff is

$$
V_{P}^{F}(\alpha, q)=\mathbb{E}_{q}\left[\frac{2 \alpha s}{(1+\alpha s)^{2}}\right]
$$

It is readily established that the low-skilled newcomer $(x)$ has more (less) effort than the better skilled rival $\left(\frac{1}{x}\right)$ when $\alpha>(<) 1$; since the equilibrium in this case is symmetric, the incumbent fights harder against the low-skilled opponent in the former case. In the case of no discrimination $(\alpha=1)$, the incumbent and both types of newcomer exert the same effort in the contest.

\subsection{Asymmetric information}

Suppose agents are asymmetrically informed. Agent $N$ knows the true value of $s$. Agent $I$ knows that the opponent is fully informed about the state, but does not know its value himself. Suppose I's belief is given by some $p \in[0,1]$, where $p=\operatorname{Pr}[s=x]$. Agents choose effort to maximize their expected payoffs: $v_{N}=\rho_{N}-e_{N}$, and $v_{I}=\mathbb{E}_{p}\left(\rho_{I}\right)-e_{I}$. As in the model of Epstein and Mealem (2013), two possibilities have to be considered, one in which both skill types are active, and one in which the low ability newcomer does not exert effort in the contest. Intuitively, when the low-skilled newcomer faces negative discrimination in the form of a low $\alpha$, the effect of his effort on the probability of success is limited, and he will prefer to exert no effort and save cost.

Lemma 2. Let $\tilde{q}=1-\frac{\alpha}{1-x}$. In the Bayes-Nash equilibrium of the asymmetric information contest, the principal's expected payoff is

$$
V_{P}^{A}(\alpha, p)= \begin{cases}2 \alpha\left(\frac{\mathbb{E}_{p}\left[\frac{1}{\sqrt{s}}\right]}{\alpha+\mathbb{E}_{p}\left[\frac{1}{s}\right]}\right)^{2} & \text { if } \alpha>1-x \cup\{1-x>\alpha \cap p \in(\tilde{q}, 1]\} \\ 2 \alpha\left(\frac{1}{x}\right)\left(\frac{1-p}{\alpha\left(\frac{1}{x}\right)+(1-p)}\right)^{2} & \text { if } 1-x>\alpha \cap p \in[0, \tilde{q}) .\end{cases}
$$

The first line in (2) represents the case in which both newcomer types exert effort in the contest; the second line occurs when only the most skilled newcomer participates, making expected effort exerted a function of only his skill level $\left(\frac{1}{x}\right)$. Note that when the discrimination parameter $\alpha \geq 1$, then both newcomer types exert effort, and this is also the case for $\alpha$ below but sufficiently close to 1 . When finally deciding on the optimal value of the discrimination parameter, the principal must bear in mind the different patterns of behavior that can ensue as documented by Lemma 2 . However, it turns out that the principal has better options in her policy setting than excluding one type of newcomer from exerting effort. Write the amount of effort exerted by the incumbent under full information when facing newcomer $s$ as $e_{I}^{F}[s]$, and the amount of effort under asymmetric information as $e_{I}^{A}$. When both types of newcomer are active, Lemmas 1 and 2 imply the following:

Corollary 1. When $\alpha>1$, then $e_{I}^{F}[x]>e_{I}^{A}>e_{I}^{F}\left[\frac{1}{x}\right]$. For $1>\alpha>1-x$, then $e_{I}^{F}\left[\frac{1}{x}\right]>e_{I}^{A}>e_{I}^{F}[x]$.

When the principal favors the newcomer, the incumbent exerts more effort against a known low-skilled opponent and is discouraged when facing the advantaged superior player. The reverse is the case when the newcomer is discriminated against; the incumbent fights intensely against the superior type, with lower effort against the already-disadvantaged low skill rival. In each case, the expected effort of the incumbent under asymmetric information lies in between the full information effort levels.

\section{Information disclosure with discrimination}

\subsection{The posterior belief}

At stage 3, nature draws a realization of the state $s \in S$ and subsequently, a signal $m \in M$ from $\pi(\cdot \mid s)$, which leads $I$ to update his belief from the prior $q$ to a posterior $q_{m} \in[0,1]$ :

$$
q_{m}=\operatorname{Pr}[s=x \mid m]=\frac{\operatorname{Pr}[m \mid s=x] \operatorname{Pr}[s=x]}{\sum_{s} \pi(m \mid s) \operatorname{Pr}(s)}=\frac{\pi(m \mid x) q}{\pi(m \mid x) q+\pi\left(m \mid \frac{1}{x}\right)(1-q)} .
$$

Observe that a posterior $q_{m}$ is a random draw from a distribution of $\pi(m \mid s)$ given some state $s$, which is stochastically drawn from a binary distribution over the state space $S=\left\{x, \frac{1}{x}\right\}$. Therefore, any set of signal distributions $\left\{\pi(\cdot \mid x), \pi\left(\cdot \mid \frac{1}{x}\right)\right\}$ 
generates a distribution of posteriors $\left\{q_{m}\right\}_{m \in M}$ with probabilities $\sum_{s \in S} \pi(m \mid s) \operatorname{Pr}(s)$. The principal's expected payoff, given the signal distributions $\left\{\pi(\cdot \mid x), \pi\left(\cdot \mid \frac{1}{x}\right)\right\}$, is

$$
\sum_{s \in S} \sum_{m \in M} V_{P}^{A}\left(\alpha, q_{m}\right) \pi(m \mid s) \operatorname{Pr}(s)
$$

\subsection{Optimal information disclosure}

At stage 2, the principal's information disclosure policy therefore solves the following problem:

$$
\max _{\pi(\cdot \mid x) \in \Delta(M), \pi\left(\cdot \mid \frac{1}{x}\right) \in \Delta(M)} \sum_{s \in S} \sum_{m \in M} V_{P}^{A}\left(\alpha, q_{m}\right) \pi(m \mid s) \operatorname{Pr}(s) \quad \text { subject to (3). }
$$

Following Kamenica and Gentzkow (2011), we reformulate (5) as a constrained optimization problem of choice over posteriors and derive the signal distributions from the optimal posteriors. While this technique is quite general, we illustrate it with an abridged proof, by constructing the signal distributions specific to our context, and it is included in the Appendix.

Lemma 3. The indirect value function of (5) is the same as the indirect value function of the following optimization problem:

$$
\begin{aligned}
\max _{\left\{q_{m} \in[0,1], \beta_{m} \in[0,1]\right\}_{m \in M}} & \sum_{m \in M} \beta_{m} V_{P}^{A}\left(\alpha, q_{m}\right) \\
\text { subject to } & \sum_{m \in M} \beta_{m}=1 \text { and } \sum_{m \in M} \beta_{m} q_{m}=q .
\end{aligned}
$$

Kamenica and Gentzkow (2011) establish that the indirect value function of (6) is well defined and given by the concave closure of the principal's expected payoff under asymmetric information. The concave closure denoted by Cav $(\alpha, q)$ is the smallest concave function that is everywhere weakly greater than $V_{P}^{A}(\alpha, q){ }^{21}$ Whether or not $V_{P}^{A}(\alpha, q)$ is strictly less than $\operatorname{Cav}(\alpha, q)$ for $q \in(0,1)$, has implication for how information is disclosed. If $V_{P}^{A}(\alpha, q)=\operatorname{Cav}(\alpha, q)$, then the maximum payoff the principal can achieve by committing to some state-conditional signal distributions is exactly the same as what she gets with no information disclosure. If instead $V_{P}^{A}(\alpha, q)<\operatorname{Cav}(\alpha, q)$, then the principal can manipulate I's belief by choosing the signal distributions suitably and improve her expected payoff from $V_{P}^{A}(\alpha, q)$ to $\operatorname{Cav}(\alpha, q)$. Proposition 1 documents the above findings. The proof is omitted; see Kamenica and Gentzkow (2011, Proposition 1, Corollaries 1 and 2) for a general analysis.

Proposition 1 (Kamenica and Gentzkow 2011). The indirect value of (6), as a function of the prior $q$, is given by Cav( $\alpha, q)$, the concave closure of $V_{P}^{A}(\alpha, q)$. The principal benefits from adjusting I's belief by disclosing information if and only if $V_{P}^{A}(\alpha, q)<$ $\operatorname{Cav}(\alpha, q)$.

We determine the principal's preferred information disclosure policy from the shape of $V_{P}^{A}(\alpha, q)$ with respect to $q$, which is summed up in Lemma 4.

Lemma 4. Define $\underline{\alpha}:=3+2 x$ and $\bar{\alpha}:=3+(2 / x)$. The following characterizes the shape of $V_{P}^{A}(\alpha, q)$ :

(a) If $0<\alpha<1$, then $V_{P}^{A}(\alpha, q)$ is decreasing in $q$. For $1>\alpha>\min \{2 x, 1-x\}, V_{P}^{A}(\alpha, q)$ is convex in $q$; for $1>\min \{2 x, 1-x\}>$ $\alpha>0, V_{P}^{A}(\alpha, q)$ is concave in $q$ for $q \in\left(0, \min \left\{\tilde{q}, q^{\prime}\right\}\right)$, and piecewise convex for $q \in\left(\min \left\{\tilde{q}, q^{\prime}\right\}, 1\right)$, where $\tilde{q}=1-\frac{\alpha}{1-x}$, $q^{\prime}=1-\frac{\alpha}{2 x}$.

(b) If $\alpha=1$, then $V_{P}^{A}(\alpha, q)$ is independent of $q$.

(c) If $1<\alpha \leq \underline{\alpha}$, then $V_{P}^{A}(\alpha, q)$ is increasing and concave in $q$.

(d) If $\underline{\alpha}<\alpha<\bar{\alpha}$, then $V_{P}^{A}(\alpha, q)$ is increasing and convex in $q$ for $q \in(0, \hat{q})$, and increasing and concave in $q$ for $q \in(\hat{q}, 1)$ where $\hat{q}:=(\alpha-\underline{\alpha}) /(\bar{\alpha}-\underline{\alpha})$.

(e) If $\bar{\alpha} \leq \alpha$, then $V_{P}^{A}(\alpha, q)$ is increasing and convex in $q$.

Fig. 1 plots $V_{P}^{A}(\alpha, q)$ against $q$ for various values of $\alpha$. For $\alpha>1-x, V_{P}^{A}(\alpha, q)$ is given by the first line of (2) and both types of newcomer are active at the contest stage. For $\alpha \in(0,1-x)$, there are four possible shapes of $V_{P}^{A}(\alpha, q)$ in part (a) of Lemma 4; the one illustrated in Fig. 1 holds for $x \in\left[\frac{1}{3}, 1\right)$, and newcomer type $x$ is inactive for $q<\tilde{q}$ and active otherwise. Fig. A.1 in the Appendix illustrates the possible shapes of $V_{P}^{A}(\alpha, q)$ for $x \in\left(0, \frac{1}{3}\right)$. In all of these cases, $V_{P}^{A}(\alpha, q)$ is decreasing in $q$, and there is a cutoff value of the prior $\tilde{q} \in(0,1)$ such that newcomer type $x$ is inactive for priors below this level, and active above. $^{22}$ The expected payoff of the principal is falling in $q$ for $1>\alpha$, and increasing for $\alpha>1$. Recall Corollary 1 ; in the former case, the incumbent fights most intensely against the highly skilled newcomer under full information, and increasing the prior on the low skilled type hence gives more and more weight to the lower effort that is expended against that rival. When $\alpha>1$, efforts are higher against the least skilled newcomer, and raising the prior belief on this type hence increases total expected effort.

\footnotetext{
${ }^{21}$ The concave closure is formally defined as follows. Fix $\alpha$. Let $\operatorname{co}\left(V_{P}^{A}(\alpha, q)\right)$ be the convex hull of the graph of $V_{P}^{A}(\alpha, q)$ as a function of $q$. Then, the concave closure is given by $\operatorname{Cav}(\alpha, q)=\sup \left\{p \mid(p, q) \in \operatorname{co}\left(V_{P}^{A}(\alpha, q)\right)\right\}$.

${ }^{22}$ We show later that the principal will not choose $\alpha$ relating to cases where one type of newcomer exerts zero effort.
} 


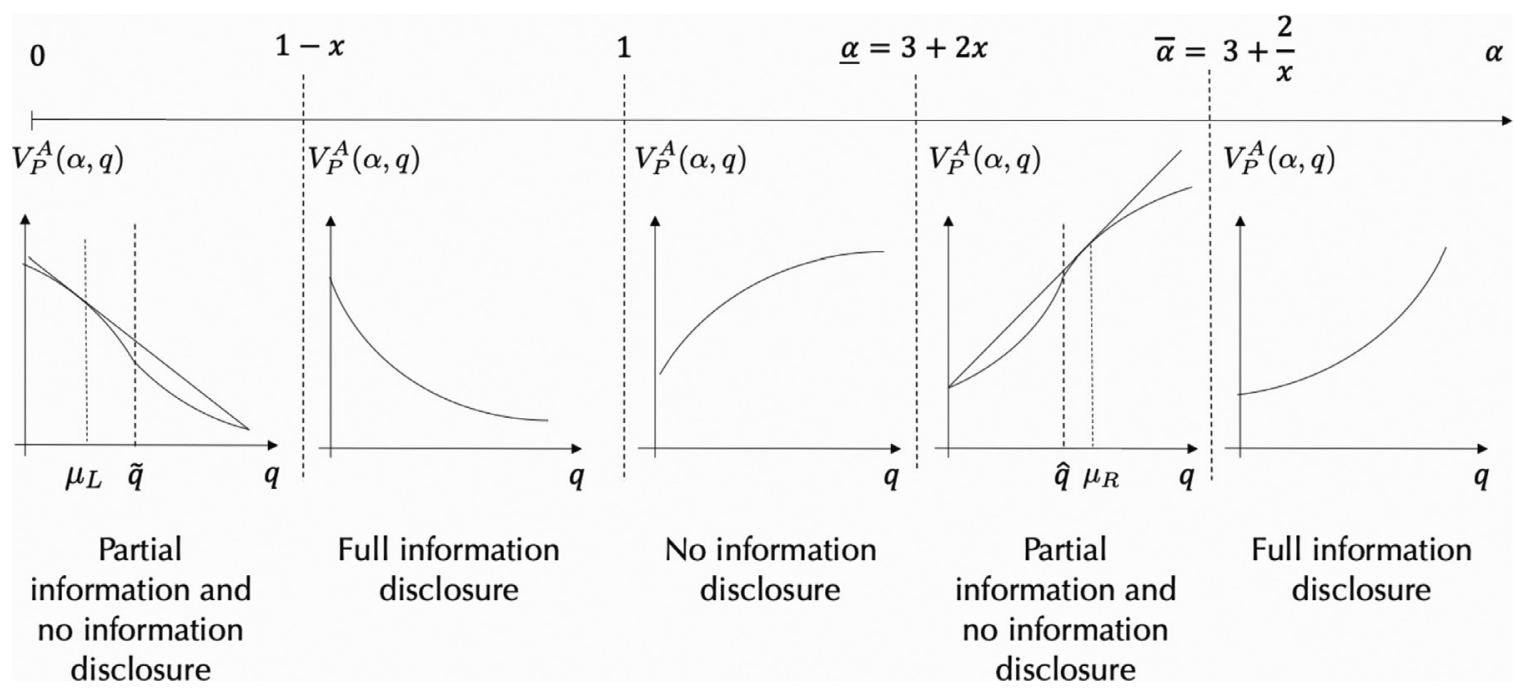

Fig. 1. Plot of $V_{P}^{A}(\alpha, q)$ against $q$ for various values of $\alpha$.

Depending on the curvature of $V_{P}^{A}(\alpha, q)$, it follows from Lemma 4 that the principal may choose one of the following three information disclosure policies in equilibrium - (i) Full information disclosure, (ii) No information disclosure, and (iii) Partial information disclosure. Below we describe the signal distributions associated with various information disclosure regimes.

First, consider Lemma 4 for $\alpha>1-x$; cases (a) and (e) are drawn in the second and fifth panels of Fig. 1 . Since $V_{P}^{A}(\alpha, q)$ is convex for all values of $q \in(0,1), \operatorname{Cav}(\alpha, q)$ is a straight line joining $V_{P}^{A}(\alpha, 0)$ and $V_{P}^{A}(\alpha, 1)$ and $V_{P}^{A}(\alpha, q)<\operatorname{Cav}(\alpha, q)$ except for $q=\{0,1\}$. The principal benefits from full information disclosure for any $q \in(0,1)$, generates a unique signal in each state so that $I$ perfectly infers the state from observing the signal. Fix a pair of signals $m_{1}, m_{2} \in M, m_{1} \neq m_{2}$; the following signal distributions generate posteriors $q_{m_{1}}=1$ and $q_{m_{2}}=0$ :

$$
\pi(m \mid x)=\left\{\begin{array}{ll}
1 & \text { if } m=m_{1} \\
0 & \text { if } m \neq m_{1}
\end{array} \text { and } \pi\left(m \mid \frac{1}{x}\right)= \begin{cases}1 & \text { if } m=m_{2} \\
0 & \text { if } m \neq m_{2}\end{cases}\right.
$$

Lemma 4, case (c), is drawn in the third panel of Fig. 1 . Since $V_{P}^{A}(\alpha, q)$ is concave for all values of $q \in(0,1), V_{P}^{A}(\alpha, q)=$ $\operatorname{Cav}(\alpha, q)$ for all $q$. No information disclosure is optimal and the principal implements this by generating one and the same signal in every state so that $I$ finds the signal uninformative. Fix some $m_{1} \in M$; the following signal distributions generate posterior $q_{m_{1}}=q$ :

$$
\pi(m \mid x)=\left\{\begin{array}{ll}
1 & \text { if } m=m_{1} \\
0 & \text { if } m \neq m_{1}
\end{array} \text { and } \pi\left(m \mid \frac{1}{x}\right)=\left\{\begin{array}{ll}
1 & \text { if } m=m_{1} \\
0 & \text { if } m \neq m_{1}
\end{array} .\right.\right.
$$

Lemma 4, case (d), is depicted in the fourth panel of Fig. 1, where $V_{P}^{A}(\alpha, q)$ is partly convex and partly concave for $q \in(0,1)$. There exists a posterior $\mu_{R} \in[\hat{q}, 1]$ such that $V_{P}^{A}(\alpha, q)<\operatorname{Cav}(\alpha, q)$ for $q \in\left(0, \mu_{R}\right)$ and $V_{P}^{A}(\alpha, q)=\operatorname{Cav}(\alpha, q)$ for $q \in\left(\mu_{R}, 1\right) .{ }^{23}$ Formally, for any $\alpha>\underline{\alpha}, \mu_{R}(\alpha)$ is defined as

$$
\mu_{R}(\alpha):=\left\{\begin{array}{ll}
p \in(0,1): \frac{V_{P}^{A}(\alpha, p)-V_{P}^{A}(\alpha, 0)}{p}=\frac{d V_{P}^{A}(\alpha, p)}{d p} & \text { if } V_{P}^{A}(\alpha, 1)-V_{P}^{A}(\alpha, 0)>\frac{d V_{P}^{A}(\alpha, 1)}{d p} \\
1 & \text { if } V_{P}^{A}(\alpha, 1)-V_{P}^{A}(\alpha, 0) \leq \frac{d V_{P}^{A}(\alpha, 1)}{d p}
\end{array} .\right.
$$

Fig. 1, panel four, illustrates that the principal implements partial information disclosure for $q \in\left(0, \mu_{R}(\alpha)\right)$ and no information disclosure for $q \in\left(\mu_{R}(\alpha), 1\right)$. For $q \in\left(0, \mu_{R}\right)$ the principal generates a common signal in both states, while randomizing with another signal in one of the two states. Fix a pair of signals $m_{1}, m_{2} \in M, m_{1} \neq m_{2}$; the following signal distributions generate posteriors $q_{m_{1}}=\mu_{R}$ and $q_{m_{2}}=0$ :

$$
\pi(m \mid x)=\left\{\begin{array}{ll}
1 & \text { if } m=m_{1} \\
0 & \text { if } m \neq m_{1}
\end{array} \text { and } \pi\left(m \mid \frac{1}{x}\right)= \begin{cases}\frac{\left(1-\mu_{R}(\alpha)\right) q}{\mu_{R}(\alpha)(1-q)} & \text { if } m=m_{1} \\
1-\frac{\left(1-\mu_{R}(\alpha)\right) q}{\mu_{R}(\alpha)(1-q)} & \text { if } m=m_{2} \\
0 & \text { if } m \notin\left\{m_{1}, m_{2}\right\}\end{cases}\right.
$$

\footnotetext{
${ }^{23}$ It is possible that $\mu_{R}=1$ so that $V_{P}^{A}(\alpha, q)$ is always strictly less than $\operatorname{Cav}(\alpha, q)$, except for $q=\{0,1\}$, so that full information disclosure is optimal.
} 
The principal also implements partial information disclosure in the first panel of Fig. 1 which illustrates one possibility from Lemma 4 , case (a). ${ }^{24}$ The posterior $\mu_{L}(\alpha)$ is given by

$$
\mu_{L}(\alpha):=\left\{\begin{array}{ll}
p \in(0,1): \frac{V_{P}^{A}(\alpha, p)-V_{P}^{A}(\alpha, 1)}{p-1}=\frac{d V_{P}^{A}(\alpha, p)}{d p} & \text { if }-\frac{d V_{P}^{A}(\alpha, 0)}{d p}<V_{P}^{A}(\alpha, 0)-V_{P}^{A}(\alpha, 1) \\
0 & \text { if }-\frac{d V_{P}^{A}(\alpha, 0)}{d p} \geq V_{P}^{A}(\alpha, 0)-V_{P}^{A}(\alpha, 1)
\end{array} .\right.
$$

No information disclosure is optimal for $q \in\left(0, \mu_{L}(\alpha)\right)$. Partial information disclosure is achieved for $q \in\left(\mu_{L}(\alpha), 1\right)$ by generating a common signal in both states, while randomizing with another signal in one of the two states. Fix a pair of signals $m_{1}, m_{2} \in M, m_{1} \neq m_{2}$; the following signal distributions generate posteriors $q_{m_{1}}=\mu_{L}$ and $q_{m_{2}}=1$ :

$$
\pi(m \mid x)=\left\{\begin{array}{ll}
\frac{\left(1-\mu_{L}(\alpha)\right) q}{\mu_{L}(\alpha)(1-q)} & \text { if } m=m_{1} \\
1-\frac{\left(1-\mu_{L}(\alpha)\right) q}{\mu_{L}(\alpha)(1-q)} & \text { if } m=m_{2} \\
0 & \text { if } m \notin\left\{m_{1}, m_{2}\right\}
\end{array} \text { and } \pi\left(m \mid \frac{1}{x}\right)= \begin{cases}1 & \text { if } m=m_{1} \\
0 & \text { if } m \neq m_{1}\end{cases}\right.
$$

Partial disclosure is no longer a possibility when $\mu_{L}(\alpha)=0$; Lemma 5 shows that this occurs for sufficiently large values of $\alpha$.

Lemma 5. There exists an $\widehat{\alpha}(x) \in(0, x)$, such that $\mu_{L}(\alpha)=0$ for $\alpha \in[\widehat{\alpha}(x), 1)$.

In Lemma 4 , case $(\mathbf{b}), V_{P}^{A}(\alpha, q)$ is independent of $q$, and any information disclosure policy yields the same payoff.

Proposition 2 characterizes the equilibrium information disclosure policy for a given degree of discrimination $\alpha$. The proof follows from Lemma 4 and the above discussion.

Proposition 2. Fix $\alpha>0, x \in(0,1)$, and $q \in(0,1)$. The equilibrium information disclosure policy is characterized as follows:

1. Suppose I is positively discriminated, i.e., $\alpha<1$.

(a) If $\alpha \in(0, \widehat{\alpha}(x))$, then the principal implements partial information disclosure with the signal distributions (12) for $q \in$ $\left(\mu_{L}(\alpha), 1\right)$, and implements no information disclosure with the signal distributions (8) for $q \in\left(0, \mu_{L}(\alpha)\right)$.

(b) If $\alpha \in[\widehat{\alpha}(x), 1)$, the principal implements full information disclosure with the signal distributions (7).

2. Suppose $N$ is positively discriminated, i.e., $\alpha>1$.

(a) If $\alpha \leq \underline{\alpha}$, then the principal implements no information disclosure with the signal distributions (8),

(b) If $\underline{\alpha}<\alpha<\bar{\alpha}$, then the principal implements partial information disclosure with the signal distributions (10) for $q \in$ $\left(0, \mu_{R}(\alpha)\right)$, and implements no information disclosure with the signal distributions (8) for $q \in\left(\mu_{R}(\alpha), 1\right)$,

(c) If $\bar{\alpha} \leq \alpha$, then the principal implements full information disclosure with the signal distributions (7).

3. Suppose no player is positively discriminated, i.e., $\alpha=1$. Then, the principal's expected payoff is invariant to any information disclosure policy.

Proposition 2 illustrates specifically how the discrimination policy determines information disclosure. When the informed newcomer is mildly discriminated against $(\alpha \in[\widehat{\alpha}(x), 1))$, it is always optimal to choose a system of signals such that the skill differential is fully disclosed. This is because the discrimination, although it reduces the effect of the skill differential when the uninformed incumbent is skill inferior, has little effect if information is not disclosed: the return to the effort of the uninformed player is then uncertain, and hence effort is risky. Information disclosure eliminates this uncertainty, encouraging the uninformed incumbent to fight even if the opponent is very skilled (see Corollary 1 ). In cases in which the informed newcomer is heavily discriminated against $(\alpha \in(0, \widehat{\alpha}(x)))$, partial disclosure is optimal since $V_{P}^{A}(\alpha, q)$ is concave in $q$ for low values of the prior, becoming convex for higher values. A highly skilled newcomer can overcome the discrimination in the contest, and when the uninformed incumbent thinks this to be very likely $\left(q \in\left(0, \mu_{L}(\alpha)\right)\right)$, the principal reveals no information since the prospect of facing a superior opponent incites the incumbent to exert effort. When the incumbent thinks it less likely that the newcomer is of high ability, the principal sends a signal allowing updating of the prior, but falling short of revealing the exact type of newcomer.

When the informed newcomer is positively discriminated, then the magnitude of discrimination is important for the optimal policy of information disclosure. With mild positive discrimination of the newcomer $(1<\alpha \leq \underline{\alpha})$, no disclosure is optimal, whilst full disclosure is best for large positive discrimination $(\bar{\alpha} \leq \alpha)$. In the former case, the players are treated almost the same, and the uninformed incumbent will exert effort as if he faces an average opponent; knowing for certain that the opponent is very skilled or little skilled is detrimental to effort since he will either be discouraged or slack off. When the discrimination is heavily in favor of the informed newcomer, the incumbent has low incentives to exert effort if he thinks he is facing an opponent of average ability; this can be alleviated to some extent by fully revealing the skill differential. For intermediate values of the discrimination parameter $(\underline{\alpha}<\alpha<\bar{\alpha})$, these concerns need to be balanced, and partial disclosure is optimal. If the uninformed incumbent thinks it very likely that the opponent is of low skill level $(q \in$ $\left.\left(\mu_{R}(\alpha), 1\right)\right)$, the principal will find it optimal not to reveal information since the incumbent fights most intensely against this rival type (Corollary 1$)$. When the incumbent thinks it likely that the opponent is of high ability $\left(q \in\left(0, \mu_{R}(\alpha)\right)\right)$, partial

\footnotetext{
24 Proposition 2 covers all cases discussed in Lemma 4, not just those drawn in Fig. 1.
} 
disclosure is optimal which involves updating of the prior belief but not full revelation of type; making the incumbent think it more likely that the newcomer is low skilled incites effort in this case.

For a given $\alpha$, the principal's expected payoff from the optimal information disclosure is Cav( $\alpha$, $q)$. We let FD and ND denote the sets of values of $\alpha$, for which the principal implements full- and no information disclosure in equilibrium, respectively. Partial discrimination can occur in two different regions for $\alpha$, and these are marked $\mathrm{PD}_{R}$ for $\alpha>1$, and $\mathrm{PD}_{L}$ for $1>\alpha$. Then 25

$$
\operatorname{Cav}(\alpha, q)= \begin{cases}V_{P}^{F}(\alpha, q) & \text { if } \alpha \in F D \\ V_{P}^{A}(\alpha, q) & \text { if } \alpha \in N D \\ \frac{q}{\mu_{R}(\alpha)} V_{P}^{A}\left(\alpha, \mu_{R}(\alpha)\right)+\left(1-\frac{q}{\mu_{R}(\alpha)}\right) V_{P}^{A}(\alpha, 0) & \text { if } \alpha \in P D_{R} \\ \frac{1-q}{1-\mu_{L}(\alpha)} V_{P}^{A}\left(\alpha, \mu_{L}(\alpha)\right)+\left(1-\frac{1-q}{1-\mu_{L}(\alpha)}\right) V_{P}^{A}(\alpha, 1) & \text { if } \alpha \in P D_{L} \\ \mathbb{E}_{q}\left[\frac{2 s}{(1+s)^{2}}\right] & \text { if } \alpha=1\end{cases}
$$

\section{Optimal discrimination}

At stage 1 , the principal's discrimination policy solves the following problem:

$$
\max _{\alpha>0} \operatorname{Cav}(\alpha, q) \text {. }
$$

Denote the solution by $\alpha^{*}$. We begin with a set of lemmas that will be useful in characterizing $\alpha^{*}$.

Lemma 6 establishes an important link between the discrimination policy and the disparity in skill. Discrimination has two effects. On the one hand, it is costly to the principal due to its asymmetric treatment of the contestants which discourages effort. On the other hand, it benefits the principal by adjusting the disparity in skill, leveling the playing field and encouraging effort. Had there been no uncertainty, the principal could perfectly mitigate the adverse effect of the skill disparity by setting $\alpha=1 / \mathrm{s}$. In the presence of uncertainty, discrimination is always costly in one state and can possibly benefit in the other, and this fact restricts the magnitude of discrimination employed by the principal.

Lemma 6. For any $q \in(0,1), \alpha^{*} \in\left[x, \frac{1}{x}\right]$.

In proving Lemma 6 we show in Lemmas A.1 and A.2 in the Appendix that it is not optimal for the principal to choose $\alpha \in P D_{L} \cup P D_{R}$ so that partial disclosure of information will not be implemented when the principal can choose discrimination level. Furthermore, we show in Lemma A.3 in the Appendix that $\alpha \in N D \cap(0, \widehat{\alpha}(x)]$ cannot be an optimal choice; specifically, we show that choosing $\alpha \in(0, \widehat{\alpha}(x)]$ and no discrimination is dominated by $\alpha=x$ combined with full information disclosure. These results allow us to eliminate cases $\mathbf{1}(\mathbf{a})$ and $\mathbf{2}(\mathbf{b})$ in Proposition 2 as candidates for optimal behavior. The former implies that the principal chooses a discrimination policy such that both newcomer types participate in the contest. The principal prefers either to remove all uncertainty relating to the state, or to keep the initial level of uncertainty in combination with an optimal discrimination parameter. A further direct implication of Lemma 6 is that the principal will not choose $\alpha>\bar{\alpha}$, a situation described in Proposition 2, case 2(c). Then only cases $\mathbf{1}(\mathbf{b})$ and $\mathbf{2}(\mathbf{a})$ are candidates for optimal behavior. Hence, we can state the following result:

Corollary 2. If $\alpha^{*} \in[x, 1)$, then the principal implements full information disclosure; if $\alpha^{*} \in\left(1, \frac{1}{x}\right]$, then the principal implements no information disclosure.

We denote the principal's optimal choice of discrimination under full and no information disclosure by $\alpha^{F D}$ and $\alpha^{N D}$. Formally,

Definition 1. $\alpha^{F D}:=\underset{\alpha>0}{\arg \max } V_{P}^{F}(\alpha, q)$ and $\alpha^{N D}:=\underset{\alpha \geq 1}{\arg \max } V_{P}^{A}(\alpha, q)$.

There will be two local maxima of $V_{P}^{A}(\alpha, q)$, depending on whether or not both newcomer types are active. Since we have already established that $\alpha^{*}$ is associated with a no-disclosure policy only when $\alpha>1$, which rules out the possibility of implementing no disclosure with only one newcomer type being active, we define $\alpha^{N D}$ as the argument of the maxima over a constrained set of $\alpha \geq 1$. Lemmas 7 and 8 characterize properties of $\alpha^{F D}$ and $\alpha^{N D}$.

Lemma 7. $\alpha^{F D}$ solves $\mathbb{E}_{q}\left[\frac{s(1-\alpha s)}{(1+\alpha s)^{3}}\right]=0$. Further, $\alpha^{F D} \lesseqgtr 1 \Leftrightarrow q \lesseqgtr \frac{1}{2}$.

Lemma 8. $\alpha^{N D}=\left\{\begin{array}{ll}\mathbb{E}_{q}\left[\frac{1}{s}\right] & \text { if } q>\frac{x}{1+x} \\ 1 & \text { if } q \leq \frac{x}{1+x}\end{array}\right.$.

We now state the main proposition, which describes the equilibrium discrimination policy.

Proposition 3. Fix $x \in(0,1)$ and $q \in(0,1)$. There exists a threshold $\bar{q} \in\left[\frac{x}{1+x}, \frac{1}{2}\right]$ such that

\footnotetext{
${ }^{25}$ For $\mathrm{PD}_{L}, V_{P}^{A}\left(\alpha, \mu_{L}(\alpha)\right)$ is evaluated using the second line of $(2)$, whilst $V_{P}^{A}(\alpha, 1)$ is evaluated using the first.
} 


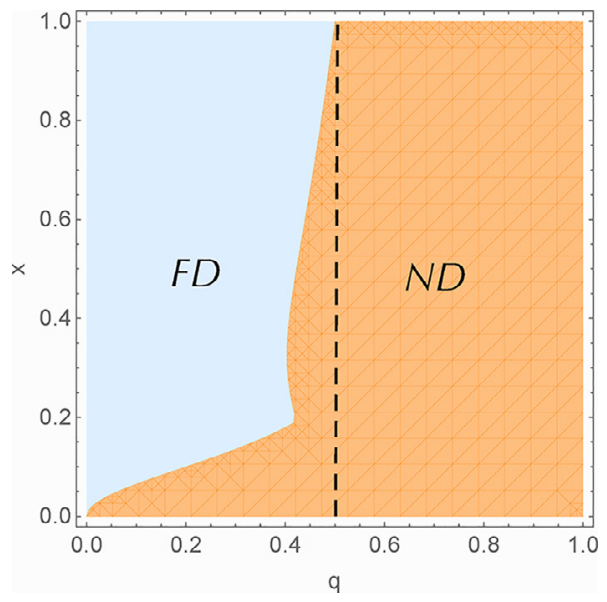

Fig. 2. The equilibrium information disclosure policy in $(q, x)$ space.

1. If $q<\bar{q}$, then the principal's choice of discrimination is $\alpha^{*}=\alpha^{F D}<1$ and there is full information disclosure in equilibrium.

2. If $q>\bar{q}$, then the principal's choice of discrimination is $\alpha^{*}=\alpha^{N D}>1$ and there is no information disclosure in equilibrium.

3. If $q=\bar{q}$, then the principal is indifferent between choosing $\alpha^{*}=\alpha^{F D}<1$ along with full information disclosure and choosing $\alpha^{*}=\alpha^{N D}>1$ along with no information disclosure.

Proposition 3 makes several important observations. Consider the principal's preference for information disclosure when she can choose the level of discrimination. Fig. 2 plots the equilibrium information disclosure policy in $(q, x)$ space. The asymmetry in information between the players can be both costly and beneficial to the principal. In the presence of asymmetric information, the return to the effort of the uninformed player $I$ is uncertain, which dampens I's incentive to raise effort. By disclosing information, the principal eliminates this uncertainty and can encourage $I$ to fight. However, when information is revealed, I comes to know the level of skill disparity in both states. A state-independent choice of discrimination has limited ability to eliminate this disparity; while discrimination may help in leveling the playing field in one state, it makes it more uneven in the other.

Recall from Corollary 2 that the benefits of not disclosing information outweigh the costs if the informed player $N$ is positively discriminated (i.e., $1<\alpha<\frac{1}{x}$ ). The principal can pursue a positive discrimination policy in favor of $N$ to her own advantage if $N$ is more likely to be skill inferior (i.e., $q>1 / 2$ ). Therefore, for $q>1 / 2$, the principal commits not to disclose information and positively discriminates $N$. Corollary 1 shows that $I$ fights most against the low-skilled newcomer in this case, and the belief that $N$ is of this type is sufficiently high that the principal chooses no disclosure. Full disclosure runs the risk of revealing a high ability type and then efforts fall for $\alpha>1$. As $q$ decreases further from $1 / 2, N$ becomes more likely to be skill superior. Positive discrimination in favor of $N$ becomes increasingly costly to the principal as $I$ reduces effort anticipating that he is more likely to be facing a stronger and positively discriminated opponent. To counter her disincentive, for sufficiently low values of $q$, the principal commits to reveal information and positively discriminate $I$ to benefit him in the unfavorable state. However, as is clear from Fig. 2, when a low value of $q$ coincides with a low value of $x$, the policy of no disclosure is optimal; here it is highly likely that the informed player has a very high skill level. Knowing this would dampen I's willingness to exert effort, and then $N$ will also slack-off.

Next, consider the principal's choice of discrimination in equilibrium. Fig. 3 plots the equilibrium discrimination $\alpha^{*}$ against $q$, for given $x=0.3$. If the principal's optimal choice of discrimination is $\mathbb{E}_{q}\left[\frac{1}{s}\right]=\alpha^{N D}$, she chooses not to disclose information. In the Appendix, (A.6) shows that the slope of $N$ 's reaction function in equilibrium is positive (negative) when $\alpha>(<) \alpha^{N D}$, so that effort is a strategic complement (substitute) for this player, making him the favorite (underdog). ${ }^{26}$ The optimal level of discrimination with no disclosure thus evens the contest, so that the marginal product of $N$ 's effort is not affected by I's effort. Similarly, when the principal sets $\alpha^{F D}$, information is fully disclosed; when the choice of discrimination parameter is made, the state is not known and this level of the discrimination parameter ensures that $\mathbb{E}_{q}\left[\frac{\partial^{2} v_{N}}{\partial e_{l} \partial e_{N}}\right]=0$, measured in equilibrium. ${ }^{27}$ Hence, neither $N$ nor $I$ is the ex ante favorite when $\alpha^{F D}$ is chosen and there is full information disclosure, implying that the contest is expected to be balanced.

\footnotetext{
${ }^{26}$ See Hurley and Shogren (1998b). The slope of $N$ 's reaction function follows the sign of $\frac{\partial^{2} v_{N}}{\partial e_{l} \partial e_{N}}$, the effect that $I$ 's effort has on the marginal product of $N$ 's own effort. If this is positive then effort is a strategic complement for $N$.

27 We have that $\frac{\partial^{2} v_{N}}{\partial e_{I} \partial e_{N}}=\alpha S\left[\frac{\alpha s e_{N}-e_{I}}{\left(\alpha s e_{N}+e_{l}\right)^{3}}\right]$. When the discrimination is set, the state is not known, so taking the expectation of this and evaluating at the symmetric equilibrium gives sign $\mathbb{E}_{q}\left[\frac{\partial^{2} v_{N}}{\partial e_{1} \partial e_{N}}\right]=\operatorname{sign} \mathbb{E}_{q}\left[\frac{s(\alpha s-1)}{(\alpha s+1)^{3}}\right]$. By Lemma $7, \alpha^{F D}$ is such that this value is zero.
} 


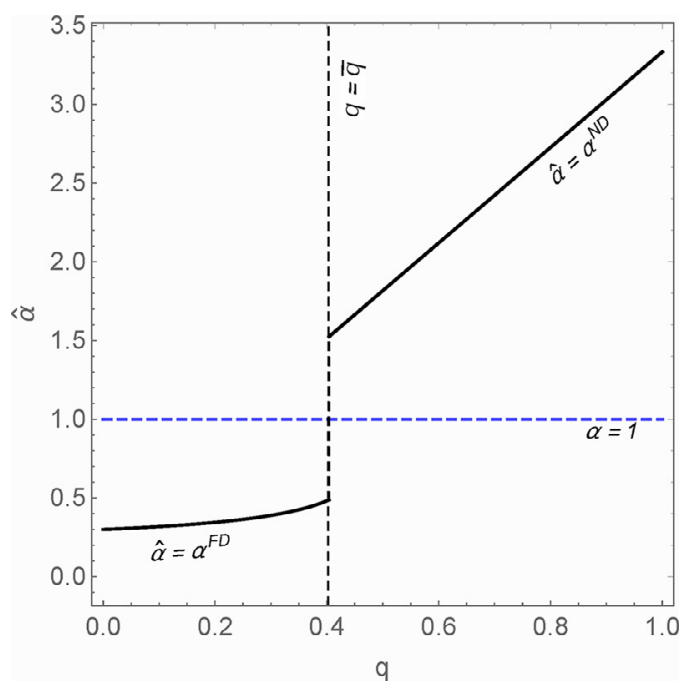

Fig. 3. The equilibrium discrimination policy $\alpha^{*}$ against $q$ (for $x=0.3$ ).

In terms of the incumbent-newcomer scenario, we consider that the newcomer is better informed of relative ability than the incumbent. Through previous play, the incumbent is likely to reveal its technological capability, physical and intellectual capacity, resource base or other attributes depending on the application. A newcomer is by definition comparatively unknown. In an innovation contest, the relative capability of a newcomer may be determined by undisclosed results of trials or prototype testing, or whether the team is suffering from innovation fatigue or burnout. In a sales contest, the leads that a salesperson has in a certain territory can contribute to relative strength; a worker external to a firm will possess qualities that are initially hidden from a potential employer compared with internal candidates.

In our model, this uncertainty is described by the two parameters $(q, x)$ capturing the likelihood that the newcomer is inferior/superior to the incumbent, and by how much. Although it is possible to set the discrimination policy in order to exclude the weaker newcomer from participation in the contest, it is not optimal to do so. Furthermore, the principal prefers to fully disclose the hidden information for many parameter combinations where $q<\frac{1}{2}$ so that the newcomer is most likely to be the superior player. Full disclosure can be a requirement to make trial or testing results publicly available in an innovation contest, or to publish territorial leads in a sales contest. Prospective external employees can be subjected to aptitude testing with results that are revealed to all. As we have seen, this policy will be combined with favorable discrimination of the incumbent in the contest. In a sales contest, the incumbent may be given better territories, less administrative duties or other resources in the contest that can boost effective effort. A newcomer in an innovation contest may be required to use administrative resources to prove that its laboratory fulfills certain requirements, provide proof of concept and/or evidence that ethical guidelines are followed. This can reduce effective effort by the newcomer in the contest. An external worker may be required to acquire knowledge of the corporate culture or business practice of the hiring firm, detracting effective effort in the contest. When the incumbent is likely to be inferior (i.e. high values of $q$ ), the principal prefers to not reveal information about relative ability, discriminating in favor of the newcomer.

\section{Alternative modeling assumptions}

The robustness of our analysis is of course dependent upon how the results would change in the light of alternative modeling assumptions. In this section, we highlight the role played by our restriction of the skill differential to $\left\{x, \frac{1}{x}\right\}$, and the multiplicative discrimination policy.

\subsection{Skill differential}

Suppose that the skill differentials are now given by $0<x<1<y$. We solve this extended model in Appendix B and our analysis shows that the main results remain intact qualitatively. This is because our model with a parameter couple $(\alpha, x)$ is isomorphic to an alternate model with a parameter triple $\left(\alpha^{\prime}, x^{\prime}, y^{\prime}\right)$ and the following mappings: $\alpha \mapsto \alpha^{\prime} \sqrt{x^{\prime} y^{\prime}}$ and $x \mapsto \frac{\sqrt{x^{\prime}}}{\sqrt{y^{\prime}}} .28$ Below we briefly discuss some of the key observations from our analysis of the extended model. First, the

\footnotetext{
${ }^{28}$ To see this, note that in our framework the relative asymmetry between the two candidates is completely captured by $\alpha s$, which takes two possible values in $\left\{\alpha x, \frac{\alpha}{x}\right\}$ in the basic model and in $\left\{\alpha^{\prime} x^{\prime}, \alpha^{\prime} y^{\prime}\right\}$ in the alternate model. The mappings $\alpha \mapsto \alpha^{\prime} \sqrt{x^{\prime} y^{\prime}}$ and $x \mapsto \frac{\sqrt{x^{\prime}}}{\sqrt{y}}$ set $\alpha x=\alpha^{\prime} x^{\prime}$ and $\frac{\alpha}{x}=\alpha^{\prime} y^{\prime}$, thus keeping effects of the distribution of $\alpha s$ similar between the two models.
} 
optimal discrimination policy is now found in the interval $\alpha^{*} \in\left[\frac{1}{y}, \frac{1}{x}\right]$. Second, the case of no discrimination ( $\left.\alpha=1\right)$ no longer delineates cases in which full or no disclosure are optimal disclosure policies as in Propositions 2 and 3 . The critical point in the extended model occurs at $\alpha=\frac{1}{\sqrt{x y}}$, which is the value of the discrimination parameter such that the expected effort is not affected by the information disclosure policy. Above (below) this level of discrimination, expected effort is increasing (decreasing) in $q$ when both types of newcomer participate. The equivalent result to Corollary 1 also sets $\alpha=\frac{1}{\sqrt{x y}}$ as the critical value of the discrimination parameter. Third, the critical value of the prior that determines the optimal policy in Proposition 3 is now given by $\bar{q}(x, y) \in\left[\frac{\sqrt{x}}{\sqrt{y}+\sqrt{x}}, \frac{1}{2}\right]$.

Whilst the three adjustments noted above can be directly recovered using the isomorphic mapping, the extended model indicates the importance of the two newcomer skill levels in relation to that of the incumbent which is normalized to 1. When $x y<(>) 1$, there is less (more) skill difference between the newcomer types than in our basic model, and the average skill level is closer (further away) to that of the incumbent. A large value of $y$, extends the range of the optimal discrimination policy at the bottom of the interval, so that heavier discrimination against the newcomer can be an optimal strategy; the chance of meeting a far superior opponent discourages effort by the incumbent if $\alpha$ is too large, and the principal dampens the impact of the newcomer's effort to mitigate this effect. When there is a small difference in skill $(x y<1)$, the interval for the optimal discrimination policy is more concentrated around 1 , and for a sufficiently low prior, the principal chooses full information disclosure and $\alpha^{F D}(x, y)$ which may now be above one. ${ }^{29}$

\subsection{Discrimination policy}

In our model, a multiplicative discrimination policy seems a natural instrument to mitigate the effect of a multiplicative skill differential. However, it can also be shown that a multiplicative discrimination policy is better than an additive discrimination policy from the principal's perspective. To see this, consider the following modification of the game in which the principal chooses an additive discrimination policy: At stage 1 of the game, the principal chooses head starts $\gamma_{N}$ and $\gamma_{I}$ so that the respective scores of $N$ and $I$ are given by $s e_{N}+\gamma_{N}$ and $e_{I}+\gamma_{I}$. Then, the success probabilities of $N$ and $I$ are $\rho_{N}=\frac{s e_{N}+\gamma_{N}}{s e_{N}+e_{I}+\gamma_{N}+\gamma_{I}}$ and $\rho_{I}=\frac{e_{I}+\gamma_{I}}{s e_{N}+e_{I}+\gamma_{N}+\gamma_{I}}$. In this modified version of the model, the Nash equilibrium of the asymmetric information contest, given the head starts $\gamma_{N}$ and $\gamma_{I}$, and that both newcomer types participate, provides the principal with an expected payoff of

$$
V_{P}^{A}\left(\gamma_{N}, \gamma_{I}, q\right)=2\left(\frac{\mathbb{E}_{q}\left[\frac{1}{\sqrt{s}}\right]}{1+\mathbb{E}_{q}\left[\frac{1}{s}\right]}\right)^{2}-\gamma_{I}-\gamma_{N} \mathbb{E}_{q}\left[\frac{1}{s}\right]
$$

The expected payoff is linear in $q .^{30}$ Therefore, the principal's expected payoff is invariant to the information structure and her optimal choices of head starts are $\gamma_{N}=\gamma_{I}=0$. Further, her maximum expected payoff is $2\left(\frac{\mathbb{E}_{q}\left[\frac{1}{\sqrt{s}}\right]}{1+\mathbb{E}_{q}\left[\frac{1}{s}\right]}\right)^{2}$, which coincides with her expected payoff from a multiplicative discrimination policy with $\alpha=1$. Our main analysis of the multiplicative discrimination policy shows that the principal's optimal multiplicative discrimination strategy involves either a positive $(\alpha>$ $1)$ or a negative $(\alpha<1)$ discrimination policy in favor of the newcomer and so any additive discrimination strategy is always suboptimal.

\section{Concluding remarks}

Competition between an incumbent and a newcomer has several defining features which we have captured in a simple model. First, the players have different levels of skills; second they have different information about the skill levels. This affects the contest success function directly, in contrast with much of the previous literature in which the rivals have asymmetric information about the value of the prize. The difference in relative skill level can be large or small, and in favor of the incumbent or the newcomer. Designing the contest to maximize effort is a challenge for the principal, which we have solved by using a combination of two policy instruments. She can commit to a signaling mechanism which may reveal - at least partially - the hidden information; she can also use a discrimination policy which treats one of the players preferentially by biasing positively his effort level in the contest. In designing the optimal policies, the principal must be mindful of the fact that the least skilled newcomer may not participate in the contest. We show that the optimal level and direction of discrimination directly determines the choice of information disclosure, and that the optimal policies induce contest effort from both types of newcomer. Further, we show how the optimal policy is connected to the prior beliefs of the uninformed player. When the uninformed player believes that the informed player is very likely to be skill-inferior, then the designer does not benefit from revealing this to the uninformed opponent, and she chooses to discriminate in favor of the informed (but likely low-skilled) player. On the other hand, when the uninformed player thinks that it is likely that the opponent

\footnotetext{
29 See Proposition B.2 in Appendix B.

30 Specifically that $V_{P}^{A}\left(\gamma_{N}, \gamma_{I}, q\right)=2 \frac{x}{(1+x)^{2}}-\gamma_{I}-\gamma_{N}\left(x+\frac{q\left(1-x^{2}\right)}{x}\right)$ in our main case.
} 
will be highly skilled, the designer must alleviate riskiness of effort for this player by revealing the true state; she will also discriminate against the informed player to encourage effort by both. The partial disclosure of the hidden information is never optimal when the discriminatory bias can be chosen optimally by the principal. Making the prior more precise is a second-best substitute for optimal discrimination. However, we show that this policy can be optimal for some given levels of the discrimination parameter when this is not under the control of the principal.

Our model captures situations in which a newcomer competes against an incumbent for a prize, and the ability of the incumbent is known by all, but the newcomer has hidden talent. The findings from our analysis can provide insights on how to design information structure to incentivize efforts in these situations. In order to get closed form solutions that allow comparison of effort levels across the whole range of the discrimination parameter, we have made some simplifying assumptions that naturally affect the generality of our results. First, we have assumed that only two players compete; this is, however, an often used construct in contest models. Second, the assumption of a two-point ability distribution is limiting, also due to the fact that the skill levels that can be realized lie either side of the ability of the uniformed player. Hence he knows that he is either facing a superior or an inferior opponent. Nevertheless, we believe that our structure is a useful first step in analyzing the potentially complex interplay between information disclosure and other policies designed to level the playing field in contest games.

\section{Declaration of Competing Interest}

We declare that we have no relevant or material financial interests that relate to the research described in this paper.

\section{Appendix A}

The Appendix contains the proofs. Further, it includes three additional lemmas which will be used in the proof of Lemma 6 and are also referred to in the discussion of Lemma 6 in the main text. It also includes an additional figure (Fig. A.1), which is referred to in the discussion of Lemma 4 and its proof.

Proof of Lemma 1. Solving the first-order payoff-maximizing conditions of both agents simultaneously. we find that the equilibrium effort under full information is symmetric:

$$
e_{N}^{F}=e_{I}^{F}=\frac{\alpha s}{(1+\alpha s)^{2}}
$$

and the expected payoffs of $N$ and $I$ are:

$$
v_{N}^{F}=\left[\frac{\alpha s}{1+\alpha s}\right]^{2}, v_{I}^{F}=\left[\frac{1}{1+\alpha s}\right]^{2} .
$$

The principal's payoff, expressed as a function of $\alpha$ and $q$, is given by

$$
V_{P}^{F}(\alpha, q)=\mathbb{E}_{q}\left[\frac{2 \alpha s}{(1+\alpha s)^{2}}\right]
$$

Proof of Lemma 2. The optimal effort of $N$ will be a function of the true value of $s$, whilst the effort of $I$ will be conditioned upon his belief. Equating the marginal benefits of effort for each agent (which equal the common marginal cost of 1 ) reveals that equilibrium efforts, $e_{N}^{A}(s), e_{I}^{A}$, satisfy

$$
\frac{\alpha s e_{I}^{A}}{\left(\alpha s e_{N}^{A}(s)+e_{I}^{A}\right)^{2}}=1=\mathbb{E}_{p}\left[\frac{\alpha s e_{N}^{A}(s)}{\left(\alpha s e_{N}^{A}(s)+e_{I}^{A}\right)^{2}}\right] .
$$

Then for any value of $s$,

$$
\alpha s e_{I}^{A}=\left(\alpha s e_{N}^{A}(s)+e_{I}^{A}\right)^{2},
$$

which further implies that

$$
\mathbb{E}_{p}\left[\frac{\alpha s e_{N}^{A}(s)}{\alpha s e_{I}^{A}}\right]=1 \Rightarrow e_{I}^{A}=\mathbb{E}_{p}\left[e_{N}^{A}(s)\right] .
$$

The first part of (A.3) implies that

$$
e_{N}^{A}(s)=\frac{\sqrt{\alpha s e_{I}^{A}}-e_{I}^{A}}{\alpha s},
$$

whereupon taking the expectation gives

$$
\mathbb{E}_{p}\left[e_{N}^{A}(s)\right]=\sqrt{e_{I}^{A}} \mathbb{E}_{p}\left[\sqrt{\frac{1}{\alpha s}}\right]-e_{I}^{A} \mathbb{E}_{p}\left[\frac{1}{\alpha s}\right] .
$$


Using the equality in (A.4) to solve (A.6) gives the final expression for the expected efforts as

$$
e_{I}^{A}=\alpha\left(\frac{\mathbb{E}_{p}\left[\frac{1}{\sqrt{s}}\right]}{\alpha+\mathbb{E}_{p}\left[\frac{1}{s}\right]}\right)^{2}=\mathbb{E}_{p}\left[e_{N}^{A}(s)\right]
$$

The principal's payoff, expressed as a function of $\alpha$ and $p$, is given by

$$
V_{P}^{A}(\alpha, p)=\mathbb{E}_{p}\left[e_{N}^{A}(s)\right]+e_{I}^{A}=2 \alpha\left(\frac{\mathbb{E}_{p}\left[\frac{1}{\sqrt{s}}\right]}{\alpha+\mathbb{E}_{p}\left[\frac{1}{s}\right]}\right)^{2}
$$

This solution is valid as long as the right-hand side in (A.5) is positive for both types. Using (A.7) in (A.5) for $s=x$, this is equivalent to $\alpha>(1-p)(1-x)$, which holds for any $p$ if $\alpha>1-x$, and otherwise for $1-x>\alpha$ and $p>1-\frac{\alpha}{1-x}:=\tilde{q}$.

When $1-x>\alpha \cap p \in[0, \tilde{q}), e_{N}^{A}(x)=0$. Then (A.4) implies $e_{I}^{A}=(1-p) e_{N}^{A}\left(\frac{1}{x}\right)$, which inserted into (A.5) gives

$$
e_{N}^{A}\left(\frac{1}{x}\right)=\frac{\alpha x(1-p)}{(\alpha+x(1-p))^{2}} .
$$

The principal's payoff is then

$$
\begin{aligned}
V_{P}^{A}(\alpha, p) & =e_{I}^{A}+(1-p) e_{N}^{A}\left(\frac{1}{x}\right)=2(1-p) e_{N}^{A}\left(\frac{1}{x}\right) \\
& =2 \alpha\left(\frac{1}{x}\right)\left(\frac{(1-p)}{\alpha\left(\frac{1}{x}\right)+(1-p)}\right)^{2}=2 \alpha x\left(\frac{(1-p)}{\alpha+x(1-p)}\right)^{2}
\end{aligned}
$$

Proof of Lemma 3. A distribution of posteriors $\left\{q_{m}\right\}_{m \in M}$ with probabilities $\beta_{m}$ is Bayes plausible if the expected value of the posterior equals the prior, i.e., $\sum_{m \in M} \beta_{m} q_{m}=q$. From (3), it follows that any set of state-conditional signal distributions generate a Bayes plausible distribution of posteriors:

$$
\sum_{m \in M} q_{m}\left(\sum_{s \in S} \pi(m \mid s) \operatorname{Pr}(s)\right)=\sum_{m \in M} \pi(m \mid x) q=q .
$$

The converse is also true - any Bayes plausible distributions of posteriors can be generated from some state-conditional signal distributions. To see this, consider a set of distributions $\left\{q_{m}\right\}_{m \in M}$ with probabilities $\beta_{m}$ such that $\sum_{m \in M} \beta_{m}=1$ and $\sum_{m \in M} \beta_{m} q_{m}=q$. Construct the state-conditional signal distributions as follows: for any given $m \in M$, define

$$
\pi(m \mid x):=\frac{\beta_{m} q_{m}}{q} \text { and } \pi\left(m \mid \frac{1}{x}\right):=\frac{\beta_{m}\left(1-q_{m}\right)}{1-q}
$$

Then, for any $s \in S, \sum_{m \in M} \pi(m \mid s)=1$. Further, these constructed conditional signal distributions generate a set of posteriors, which is the same as $\left\{q_{m}\right\}_{m \in M}$ :

$$
\begin{aligned}
\operatorname{Pr}[x \mid m] & =\frac{\operatorname{Pr}[m \mid s=x] \operatorname{Pr}[s=x]}{\operatorname{Pr}[m \mid s=x] \operatorname{Pr}[s=x]+\operatorname{Pr}\left[m \mid s=\frac{1}{x}\right] \operatorname{Pr}\left[s=\frac{1}{x}\right]} \\
& =\frac{\frac{\beta_{m} q_{m}}{q} \cdot q}{\frac{\beta_{m} q_{m}}{q} \cdot q+\frac{\beta_{m}\left(1-q_{m}\right)}{1-q} \cdot(1-q)}=q_{m} .
\end{aligned}
$$

Therefore, the indirect value function of (5), in which $P$ maximizes her expected payoff over all possible state-conditional signal distributions, is the same as the indirect value function of the following optimization problem, in which $P$ maximizes her expected payoff over all Bayes plausible distribution of posteriors:

$$
\begin{aligned}
\max _{\left\{q_{m} \in[0,1], \beta_{m} \in[0,1]\right\}_{m \in M}} & \sum_{m \in M} \beta_{m} V_{P}^{A}\left(\alpha, q_{m}\right) \\
\text { subject to } & \sum_{m \in M} \beta_{m}=1 \text { and } \sum_{m \in M} \beta_{m} q_{m}=q .
\end{aligned}
$$

Proof of Lemma 4. From (2), distinguish the case in which both newcomer types are active as $\hat{V}_{P}^{A}(\alpha, q)=2 \alpha\left(\frac{\mathbb{E}_{q}\left[\frac{1}{\sqrt{s}}\right]}{\alpha+\mathbb{E}_{q}\left[\frac{1}{s}\right]}\right)^{2}$, and the case in which newcomer type $x$ is inactive as $\tilde{V}_{P}^{A}(\alpha, q)=2 \alpha x\left(\frac{1-q}{\alpha+x(1-q)}\right)^{2}$. Consider the first- and the second-order 


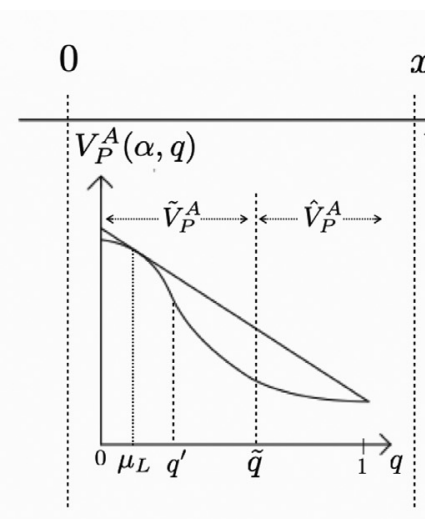

Partial information and no information disclosure

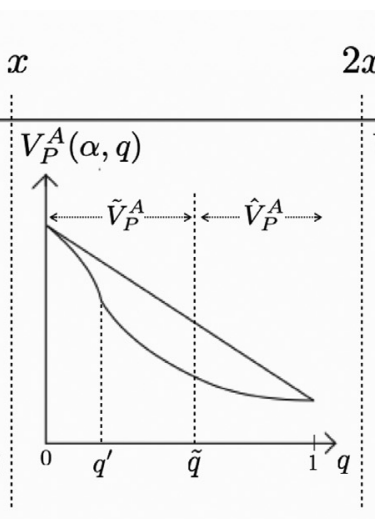

Full information disclosure

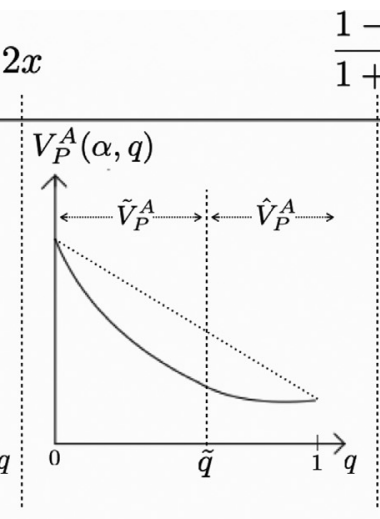

Full information disclosure $1-x$

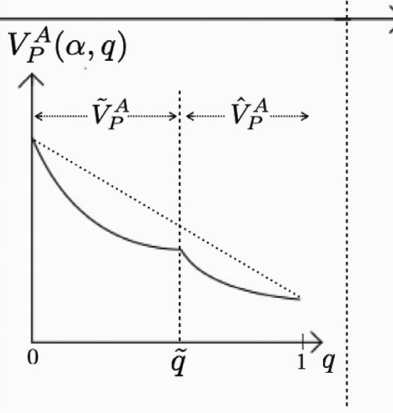

Full

information disclosure

Fig. A.1. Plot of $V_{P}^{A}(\alpha, q)$ against $q$ for $\alpha \in(0,1-x), x \in\left(0, \frac{1}{3}\right)$.

derivatives of $\widehat{V}_{P}^{A}(\alpha, q)$ and $\widetilde{V}_{P}^{A}(\alpha, q)$ with respect to $q$ :

$$
\begin{aligned}
& \frac{d \hat{V}_{P}^{A}(\alpha, q)}{d q}=\frac{4 \alpha(\alpha-1)\left(\frac{1}{\sqrt{x}}-\sqrt{x}\right) \mathbb{E}_{q}\left[\frac{1}{\sqrt{s}}\right]}{\left(\alpha+\mathbb{E}_{q}\left[\frac{1}{s}\right]\right)^{3}}, \\
& \frac{d^{2} \hat{V}_{P}^{A}(\alpha, q)}{d q^{2}}=\frac{4 \alpha(\alpha-1)\left(\frac{1}{\sqrt{x}}-\sqrt{x}\right)^{2}\left(\alpha-\left(3+2 \mathbb{E}_{q}\left[\frac{1}{s}\right]\right)\right)}{\left(\alpha+\mathbb{E}_{q}\left[\frac{1}{s}\right]\right)^{4}} . \\
& \frac{d \widetilde{V}_{P}^{A}(\alpha, q)}{d q}=-\frac{4 \alpha^{2} x(1-q)}{(\alpha+x(1-q))^{3}}, \\
& \frac{d^{2} \widetilde{V}_{P}^{A}(\alpha, q)}{d q^{2}}=\frac{4 \alpha^{2} x(\alpha-2 x(1-q))}{(\alpha+x(1-q))^{4}} .
\end{aligned}
$$

From (A.11), it follows that $\widehat{V}_{P}^{A}(\alpha, q)$ is increasing, decreasing, and invariant with respect to $q$ if $\alpha$ is greater than, less than, and equal to 1 , respectively. From (A.13), $\widetilde{V}_{P}^{A}(\alpha, q)$ is decreasing in $q$.

Consider $\alpha<1$. From (A.12), we find that $\frac{d^{2} \widehat{V}_{P}^{A}(\alpha, q)}{d q^{2}}>0$ for all $q \in(0,1)$, implying that $V_{P}^{A}(\alpha, q)$ is convex for $1>\alpha>$ $1-x$ or $1-x>\alpha$ and $q \in(\tilde{q}, 1)$, i.e. for the values of the prior for which both newcomer types exert effort. From (A.14), it is evident that $\widetilde{V}_{P}^{A}(\alpha, q)$ is convex in $q$ for $1>\alpha>2 x$. Taken together, these observations imply that $V_{P}^{A}(\alpha, q)$ is convex in $q$ for $1>\alpha>\min \{2 x, 1-x\}$.

Consider next $1>\min \{2 x, 1-x\}>\alpha>0$. We have already established that when $\widehat{V}_{P}^{A}(\alpha, q)$ represents the principal's payoff, it is convex in $q$ for $q \in(\tilde{q}, 1)$. When the principal's payoff is given by $\widetilde{V}_{P}^{A}(\alpha, q)$, it is concave in $q$ for $\alpha-2 x(1-q)<0$, i.e. for $q \in\left(0, q^{\prime}\right)$, and convex otherwise, i.e. $q \in\left(q^{\prime}, 1\right)$. Then, for low values of $q, V_{P}^{A}(\alpha, q)$ is given by $\widetilde{V}_{P}^{A}(\alpha, q)$ and it is concave; this occurs for $q \in\left(0, \min \left\{\tilde{q}, q^{\prime}\right\}\right)$. If $\tilde{q}<q^{\prime}$, then $V_{P}^{A}(\alpha, q)=\widetilde{V}_{P}^{A}(\alpha, q)$ for $q \in(0, \tilde{q})$, and it is concave in $q$. For $q \in(\tilde{q}, 1)$, then $V_{P}^{A}(\alpha, q)=\widehat{V}_{P}^{A}(\alpha, q)$, which we have shown to be convex in $q$. If, on the other hand, $\tilde{q}>q^{\prime}$, then $V_{P}^{A}(\alpha, q)=\widetilde{V}_{P}^{A}(\alpha, q)$ for $q \in(0, \tilde{q})$, and it is concave in $q$ for $q \in\left(0, q^{\prime}\right)$ and convex in $q$ for $q \in\left(q^{\prime}, \tilde{q}\right)$. As before, when $q \in(\tilde{q}, 1)$, then $V_{P}^{A}(\alpha, q)=\widehat{V}_{P}^{A}(\alpha, q)$, which is convex in $q$.

Next, consider $\alpha>1$, in which case $V_{P}^{A}(\alpha, q)=\widehat{V}_{P}^{A}(\alpha, q)$. From (A.12), we find that $\frac{d^{2} V_{P}^{A}(\alpha, q)}{d q^{2}}<0$ for all $q \in(0,1)$ if $\alpha \leq 3+2 x, \frac{d^{2} V_{P}^{A}(\alpha, q)}{d q^{2}}>0$ for all $q \in(0,1)$ if $\alpha \geq 3+\frac{2}{x}$, and $\frac{d^{2} V_{P}^{A}(\alpha, q)}{d q^{2}} \gtreqless 0$ for all $q \lesseqgtr \frac{\alpha-3-2 x}{2\left(\frac{1}{x}-x\right)}$ if $3+2 x<\alpha<3+\frac{2}{x}$. Therefore, $V_{P}^{A}(\alpha, q)$ is concave if $\alpha \leq \underline{\alpha}=3+2 x$, convex if $\alpha \geq \bar{\alpha}=3+\frac{2}{x}$, and convex (concave) in $q$ for $q \in(0, \hat{q})$ (for $q \in(\hat{q}, 1)$ ) where $\hat{q}=\frac{\alpha-3-2 x}{2\left(\frac{1}{x}-x\right)}=\frac{\alpha-\underline{\alpha}}{\alpha-\underline{\alpha}}$.

Fig. A.1 illustrates the cases from Lemma 4 that are not covered in Fig. 1. The first panel in Fig. A.1 is similar to the first panel in Fig. 1, since there is no information disclosure for low values of $q$, and partial discrimination above the threshold level $\mu_{L}$. The difference between the two cases is that $\widetilde{V}(\alpha, q)$ has an inflection point at $q^{\prime}<\tilde{q}$ in Fig. A.1, but the 
inflection occurs at $q^{\prime}>\tilde{q}$ in the first panel of Fig. 1 , by which time $\widehat{V}_{P}^{A}(\alpha, q)$ has become the expression for the expected payoff of the principal. The other cases in Fig. A.1 disappear if $2 x>1-x$, i.e. $x>\frac{1}{3}$. The expected payoff of the principal is continuous at $q=\tilde{q}$, but not differentiable. The relative slope of the function to the left and right of this point is what distinguishes these two cases. Full disclosure is optimal in each case since the straight line between the extreme points lies always above the curves. To see this for the case in the third panel of Fig. A.1, we have that the straight line is given by $q \widehat{V}(\alpha, 1)+(1-q) \widetilde{V}(\alpha, 0)$, which measured at $\tilde{q}$ gives $2 \alpha x\left(\frac{x^{2}+x \alpha\left(3-\alpha^{2}\right)+\alpha\left(1+\alpha-\alpha^{2}\right)}{(\alpha x+1)^{2}(\alpha+x)^{2}}\right)$. Furthermore, $\widehat{V}(\alpha, \tilde{q})=\widetilde{V}(\alpha, \tilde{q})=2 \alpha x$. Direct calculation gives

$$
\begin{aligned}
& 2 \alpha x\left(\frac{x^{2}+x \alpha\left(3-\alpha^{2}\right)+\alpha\left(1+\alpha-\alpha^{2}\right)}{(\alpha x+1)^{2}(\alpha+x)^{2}}\right)-2 \alpha x \\
= & 2 \alpha x(1-\alpha-x)\left(\frac{\alpha x^{3}+\left(\alpha^{2}+\alpha+2\right) x^{2}+(3 \alpha+2) x+(\alpha+1)}{(\alpha x+1)^{2}(\alpha+x)^{2}}\right)>0 .
\end{aligned}
$$

Note that $1-\alpha-x>0$ in the area covered by this cases since $\tilde{q}>0$ and $\tilde{q}=1-\frac{\alpha}{1-x}=\frac{1-\alpha-x}{1-x}>0$.

Proof of Lemma 5. Solving $-\frac{d V_{P}^{A}(\alpha, 0)}{d p} \geq V_{P}^{A}(\alpha, 0)-V_{P}^{A}(\alpha, 1)$ gives $\alpha \geq \widehat{\alpha}(x)$, where

$$
\widehat{\alpha}(x)=B-\frac{x\left(5-x^{2}\right)}{3\left(x^{2}+1\right)}+\frac{\left(x^{6}-13 x^{4}+19 x^{2}-3\right)}{9 B\left(x^{2}+1\right)^{2}}
$$

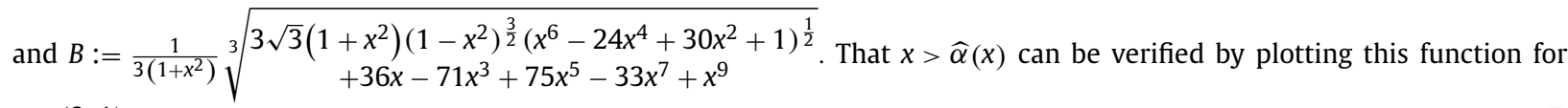
$x \in(0,1)$.

To prove Lemma 6, it is first necessary to establish that $\alpha \in P D_{L} \cup P D_{R}$ and $\alpha \in N D \cap(0, \widehat{\alpha}(x)]$ are not optimal choices. This is done in the next three lemmas (Lemma A.1, Lemma A.2, and Lemma A.3).

Lemma A.1. Consider $\underline{\alpha}<\frac{1}{x}$ and $\alpha \in P D_{R} \cap(\underline{\alpha}, \infty)$. Then, the principal's expected payoff from partial information disclosure is less than her expected payoff from no information disclosure with $\alpha=\underline{\alpha}$.

Proof. Consider $\alpha \in P D_{R} \cap(\underline{\alpha}, \infty)$ and $q \in\left(0, \mu_{R}(\alpha)\right)$. From (9), direct calculation gives

$$
\mu_{R}(\alpha)=\min \left\{\frac{x(\alpha-\underline{\alpha})(x+\alpha)}{\left(1-x^{2}\right)(1+2 x+\alpha)}, 1\right\}
$$

Let $V_{P}^{P D_{R}}(\alpha, q)$ denote the principal's expected payoff under partial information disclosure. Further, by Proposition 2, for all $\alpha \in P D_{R}$,

$$
V_{P}^{P D_{R}}(\alpha, q)=\operatorname{Cav}(\alpha, q)
$$

Since $\operatorname{Cav}(\alpha, q)$ is the indirect value function of (A.10), we apply the envelope theorem to get

$$
\frac{d \operatorname{Cav}(\alpha, q)}{d \alpha}=\sum_{m \in M} \widehat{\beta}_{m} \frac{\partial V_{P}^{A}\left(\alpha, \widehat{q}_{m}\right)}{\partial \alpha},
$$

where $\widehat{\beta}_{m} \in[0,1]$ and $\widehat{q}_{m} \in[0,1]$ are solutions of $\left(\right.$ A.10). Note that for all $\alpha \in P D_{R}$, these solutions are given by: $\widehat{\beta}_{1}=$ $\frac{q}{\mu_{R}(\alpha)}, \widehat{\beta}_{2}=1-\frac{q}{\mu_{R}(\alpha)} ; \widehat{q}_{1}=\mu_{R}(\alpha), \widehat{q}_{2}=0$. Therefore, for $\alpha \in P D_{R}$, we have

$$
\begin{aligned}
\frac{d V_{P}^{P D_{R}}(\alpha, q)}{d \alpha} & =\frac{d \operatorname{Cav}(\alpha, q)}{d \alpha}=\widehat{\beta}_{1} \frac{\partial V_{P}^{A}\left(\alpha, \widehat{q}_{1}\right)}{\partial \alpha}+\widehat{\beta}_{2} \frac{\partial V_{P}^{A}\left(\alpha, \widehat{q}_{2}\right)}{\partial \alpha} \\
& =\frac{q}{\mu_{R}(\alpha)} \frac{\partial V_{P}^{A}\left(\alpha, \mu_{R}(\alpha)\right)}{\partial \alpha}+\left(1-\frac{q}{\mu_{R}(\alpha)}\right) \frac{\partial V_{P}^{A}(\alpha, 0)}{\partial \alpha}
\end{aligned}
$$

We show that both $\frac{\partial V_{P}^{A}\left(\alpha, \mu_{R}(\alpha)\right)}{\partial \alpha}$ and $\frac{\partial V_{P}^{A}(\alpha, 0)}{\partial \alpha}$ are negative for $\alpha \in P D_{R} \cap(\underline{\alpha}, \infty)$.

From (A.18), $\frac{\partial V_{P}^{A}(\alpha, 0)}{\partial \alpha}=\frac{2 \mathbb{E}_{p=0}^{2}\left[\frac{1}{\sqrt{s}}\right][x-\alpha]}{\left(\alpha+\mathbb{E}_{p=0}\left[\frac{1}{s}\right]\right)^{3}}<0$, since $x<\underline{\alpha}<\alpha$.

Further, from (A.18), $\frac{\partial V_{P}^{A}\left(\alpha, \mu_{R}(\alpha)\right)}{\partial \alpha}=\frac{2 \mathbb{E}_{\mu_{R}(\alpha)}^{2}\left[\frac{1}{\sqrt{s}}\right]\left[\mathbb{E}_{\mu_{R}(\alpha)}\left[\frac{1}{s}\right]-\alpha\right]}{\left(\alpha+\mathbb{E}_{\mu_{R}(\alpha)}\left[\frac{1}{s}\right]\right)^{3}}$, which is negative if and only if $\left[\mathbb{E}_{\mu_{R}(\alpha)}\left[\frac{1}{s}\right]-\alpha\right]$ is negative.

Note that

$$
\mathbb{E}_{\mu_{R}(\alpha)}\left[\frac{1}{s}\right]-\alpha=x+\mu_{R}(\alpha)\left(\frac{1}{x}-x\right)-\alpha=x+\frac{(\alpha-\underline{\alpha})(x+\alpha)}{(1+2 x+\alpha)}-\alpha
$$




$$
=\frac{(x+\alpha)(x-\underline{\alpha})+(1+x)(x-\alpha)}{(1+2 x+\alpha)},
$$

which is negative since $x<\underline{\alpha}<\alpha$.

Hence, we conclude that $\frac{d V_{P}^{P D_{R}}(\alpha, q)}{d \alpha}$ is negative for $\alpha \in P D_{R} \cap(\underline{\alpha}, \infty)$. Further, since at $\alpha=\underline{\alpha}, \operatorname{Cav}(\alpha, q)=V_{P}^{A}(\alpha, q)$ and it is continuous, we conclude that the principal's expected payoff from partial information disclosure is less than her expected payoff from no information disclosure with $\alpha=\underline{\alpha}$.

Lemma A.2. Consider $\alpha \in P D_{L} \cap(0, \hat{\alpha}(x))$. Then, the principal's expected payoff from partial information disclosure is less than her expected payoff from full information disclosure with $\alpha=x$.

Proof. Consider $\alpha \in P D_{L} \cap(0, \hat{\alpha}(x))$ and $q \in\left(\mu_{L}(\alpha), 1\right)$. Note that for $\alpha \in[\widehat{\alpha}(x), 2 x], \quad \mu_{L}(\alpha) \leq 0$ and that $V_{P}^{F}(q, \alpha) \geq$ $\frac{1-q}{1-\mu_{L}(\alpha)} V_{P}^{A}\left(\alpha, \mu_{L}(\alpha)\right)+\left(1-\frac{1-q}{1-\mu_{L}(\alpha)}\right) V_{P}^{A}(\alpha, 1)$ for all $q \in[0,1]$. We can show that any choice of $\alpha \in P D_{L}$ gives the principal a lower expected payoff than choosing $\alpha=x$, which by Proposition 2 leads to full disclosure of information and the payoff $V_{P}^{F}(x, q)$. Then $V_{P}^{F}(x, 1)=\frac{2 x^{2}}{\left(1+x^{2}\right)^{2}}>\frac{2 \alpha x}{(\alpha x+1)^{2}}=V_{P}^{A}(\alpha, 1)$ since $\alpha^{P D_{L}}<x$. For $q<\tilde{q}, V_{P}^{A}(\alpha, q)=2 \alpha x\left(\frac{1-q}{\alpha+x(1-q)}\right)^{2}$ which is a concave function in $\alpha$, with a maximum at $\alpha=x(1-q)$. The maximum value of this function, measured at $q=\mu_{L}$ is $V_{P}^{A}\left(x(1-q), \mu_{L}\right)=\frac{1-\mu_{L}}{2}$. Furthermore, $V_{P}^{F}\left(x, \mu_{L}\right)-V_{P}^{A}\left(x(1-q), \mu_{L}\right)=2 \mu_{L} \frac{x^{2}}{\left(1+x^{2}\right)^{2}}>0$. Since $V_{P}^{F}(x, q)=q V_{P}^{A}(x, 1)+$ $(1-q) V_{P}^{A}(x, 0)$ and $\frac{1-q}{1-\mu_{L}(\alpha)} V_{P}^{A}\left(\alpha, \mu_{L}(\alpha)\right)+\left(1-\frac{1-q}{1-\mu_{L}(\alpha)}\right) V_{P}^{A}(\alpha, 1)$ both represent straight lines in $(V, q)$ space, and two points on the former are always strictly above the latter, it follows that setting $\alpha=x$ with full disclosure can always achieve a larger expected payoff than selecting $\alpha \in P D_{L}$ with partial disclosure.

Lemma A.3. Consider $\alpha \in N D \cap(0, \hat{\alpha}(x))$. Then, the principal's expected payoff from no information disclosure is less than her expected payoff from full information disclosure with $\alpha=x$.

Proof. Consider $\alpha \in N D \cap(0, \hat{\alpha}(x))$ and $q \in\left(0, \mu_{L}(\alpha)\right)$. In this region, $\operatorname{Cav}(\alpha, q)=V_{P}^{A}(\alpha, q)=2 \alpha x\left(\frac{1-q}{\alpha+x(1-q)}\right)^{2}$, and Lemma A.2 established that this is maximized for $\alpha=x(1-q)$, with maximum value $V_{P}^{A}(x(1-q), q)=\frac{1-q}{2}$. A policy of no information disclosure is followed by the principal for $q \in\left(0, \mu_{L}\right)$. Suppose that the principal sets $\alpha=x$, and follows a full disclosure policy. The expected payoff is given by $V_{P}^{F}(x, q)=q V_{P}^{A}(x, 1)+(1-q) V_{P}^{A}(x, 0)=q V_{P}^{A}(x, 1)+\frac{1-q}{2}$, since $V_{P}^{A}(x, 0)=\frac{1}{2}$. Recall that $V_{P}^{A}(x, 1)$ is given by $\widehat{V}_{P}^{A}(x, 1)>0$. Hence $V_{P}^{F}(x, q)>\frac{1-q}{2}$, and full disclosure with $\alpha=x$ gives the principal a higher expected payoff than setting $\alpha \in N D \cap(0, \hat{\alpha}(x))$ and no disclosure.

Proof of Lemma 6. Recall that $\operatorname{Cav}(\alpha, q)$ is the indirect value function of (A.10). Assume first that $\alpha>1$. Lemma A.1 establishes that any optimal choice in this region must not involve partial information disclosure. Applying the envelope theorem, we get that

$$
\frac{d \operatorname{Cav}(\alpha, q)}{d \alpha}=\sum_{m \in M} \widehat{\beta}_{m} \frac{\partial V_{P}^{A}\left(\alpha, \widehat{q}_{m}\right)}{\partial \alpha},
$$

where $\widehat{\beta}_{m} \in[0,1]$ and $\widehat{q}_{m} \in[0,1]$ are solutions of (A.10). Further, for any arbitrary distribution $p \in[0,1]$,

$$
\frac{\partial V_{P}^{A}(\alpha, p)}{\partial \alpha}=\frac{\partial}{\partial \alpha} 2 \alpha\left(\frac{\mathbb{E}_{p}\left[\frac{1}{\sqrt{s}}\right]}{\alpha+\mathbb{E}_{p}\left[\frac{1}{s}\right]}\right)^{2}=2 \mathbb{E}_{p}^{2}\left[\frac{1}{\sqrt{s}}\right] \frac{\partial}{\partial \alpha}\left[\frac{\alpha}{\left(\alpha+\mathbb{E}_{p}\left[\frac{1}{s}\right]\right)^{2}}\right]=\frac{2 \mathbb{E}_{p}^{2}\left[\frac{1}{\sqrt{s}}\right]\left[\mathbb{E}_{p}\left[\frac{1}{s}\right]-\alpha\right]}{\left(\alpha+\mathbb{E}_{p}\left[\frac{1}{s}\right]\right)^{3}},
$$

which is strictly positive for $\alpha<\mathbb{E}_{p}\left[\frac{1}{s}\right]$ and strictly negative for $\alpha>\mathbb{E}_{p}\left[\frac{1}{s}\right]$. Since, for any $\widehat{q}_{m} \in[0,1], \mathbb{E}_{\widehat{q}_{m}}\left[\frac{1}{s}\right] \in\left[x, \frac{1}{x}\right]$, (A.17) is strictly positive for $\alpha<x$ and strictly negative for $\alpha>\frac{1}{x}$. Hence, the solution of (14) lies in $\left(1, \frac{1}{x}\right.$ ].

Suppose now that $1>\alpha$. Lemmas A.2 and A.3 establish that any optimal choice in this region must involve full disclosure and hence the expected payoff of the principal is $V_{P}^{F}(\alpha, p)$. For any arbitrary distribution $p \in[0,1]$,

$$
\frac{\partial V_{P}^{F}(\alpha, p)}{\partial \alpha}=2 \mathbb{E}_{p}\left[\frac{s(1-\alpha s)}{(1+\alpha x)^{3}}\right]
$$

which is strictly positive for $\alpha \in\left[x, \frac{1}{x}\right]$. Thus the optimal policy in this region lies in the interval $[x, 1)$.

Combining results from both intervals shows that $\alpha^{*} \in\left[x, \frac{1}{x}\right]$.

Proof of Lemma 7. Consider the derivative of $V_{P}^{F}(\alpha, q)$ with respect to $\alpha$ :

$$
\frac{d V_{P}^{F}(\alpha, q)}{d \alpha}=\frac{d}{d \alpha} \mathbb{E}_{q}\left[\frac{2 \alpha s}{(1+\alpha s)^{2}}\right]=\mathbb{E}_{q}\left[\frac{d}{d \alpha} \frac{2 \alpha s}{(1+\alpha s)^{2}}\right]=\mathbb{E}_{q}\left[\frac{2 s(1-\alpha s)}{(1+\alpha s)^{3}}\right] .
$$

Therefore, $\alpha^{F D}$ solves (A.19). To prove the next step, the following claims will be useful. 
Claim 1. $V_{P}^{F}(\alpha, q)=V_{P}^{F}\left(\frac{1}{\alpha}, 1-q\right)$.

Proof of Claim 1. From (1),

$$
\begin{aligned}
V_{P}^{F}\left(\frac{1}{\alpha}, 1-q\right) & =\mathbb{E}_{1-q}\left[\frac{2 \frac{s}{\alpha}}{\left(1+\frac{s}{\alpha}\right)^{2}}\right]=\mathbb{E}_{1-q}\left[\frac{2 \frac{\alpha}{s}}{\left(\frac{\alpha}{s}+1\right)^{2}}\right] \\
& =(1-q)\left[\frac{2 \frac{\alpha}{x}}{\left(\frac{\alpha}{x}+1\right)^{2}}\right]+q\left[\frac{2 \alpha x}{(\alpha x+1)^{2}}\right]=\mathbb{E}_{q}\left[\frac{2 \alpha s}{(1+\alpha s)^{2}}\right]=V_{P}^{F}(\alpha, q)
\end{aligned}
$$

Claim 2. $V_{P}^{F}(\alpha, q) \gtreqless V_{P}^{F}(\alpha, 1-q) \Leftrightarrow(2 q-1)\left(\alpha^{2}-1\right) \gtreqless 0$.

Proof of Claim 2: From (1),

$$
\begin{aligned}
V_{P}^{F}(\alpha, q)-V_{P}^{F}(\alpha, 1-q) & =\mathbb{E}_{q}\left[\frac{2 \alpha s}{(1+\alpha s)^{2}}\right]-\mathbb{E}_{1-q}\left[\frac{2 \alpha s}{(1+\alpha s)^{2}}\right] \\
& \left.\left.=q\left[\frac{2 \alpha x}{(\alpha x+1)^{2}}\right]+(1-q)\left[\frac{2 \frac{\alpha}{x}}{\left(\frac{\alpha}{x}+1\right)^{2}}\right]-(1-q)\left[\frac{2 \alpha x}{(\alpha x+1)^{2}}\right]-q\right] \frac{2 \frac{\alpha}{x}}{\left(\frac{\alpha}{x}+1\right)^{2}}\right] \\
& =(1-2 q)\left[\frac{2 \frac{\alpha}{x}}{\left(\frac{\alpha}{x}+1\right)^{2}}-\frac{2 \alpha x}{(\alpha x+1)^{2}}\right]=(1-2 q)\left[\frac{2 \alpha x}{(\alpha+x)^{2}}-\frac{2 \alpha x}{(\alpha x+1)^{2}}\right] \\
& =\frac{2 \alpha x(1-2 q)}{(\alpha+x)^{2}(\alpha x+1)^{2}}\left[(\alpha x+1)^{2}-(\alpha+x)^{2}\right]=\frac{2 \alpha x(1-2 q)\left(1-\alpha^{2}\right)\left(1-x^{2}\right)}{(\alpha+x)^{2}(\alpha x+1)^{2}}
\end{aligned}
$$

Since $x \in(0,1), V_{P}^{F}(\alpha, q) \gtreqless V_{P}^{F}(\alpha, 1-q) \Leftrightarrow(1-2 q)\left(1-\alpha^{2}\right) \gtreqless 0$. This completes the proof of Claim 2 .

From Claim 2, replacing $\alpha$ by $\frac{1}{\alpha}$ and $q$ by $1-q$, we get

$$
V_{P}^{F}\left(\frac{1}{\alpha}, 1-q\right) \gtreqless V_{P}^{F}\left(\frac{1}{\alpha}, q\right) \Leftrightarrow(2 q-1)\left(\alpha^{2}-1\right) \gtreqless 0 .
$$

By Claim 1, it follows then

$$
V_{P}^{F}(\alpha, q) \gtreqless V_{P}^{F}\left(\frac{1}{\alpha}, q\right) \Leftrightarrow(2 q-1)\left(\alpha^{2}-1\right) \gtreqless 0 \Leftrightarrow\left(q-\frac{1}{2}\right)(\alpha-1) \gtreqless 0 .
$$

Note that by definition of $\alpha^{F D}, V_{P}^{F}\left(\alpha^{F D}, q\right) \geq V_{P}^{F}\left(\frac{1}{\alpha^{F D}}, q\right)$. Therefore, from (A.20), we get $\left(q-\frac{1}{2}\right)\left(\alpha^{F D}-1\right) \geq 0$, and equivalently, $\alpha^{F D} \lesseqgtr 1 \Leftrightarrow q \lesseqgtr \frac{1}{2}$.

Proof of Lemma 8. Note that for $\alpha \geq 1$, both newcomer types are active and $V_{P}^{A}(\alpha, q)=2 \alpha\left(\frac{\mathbb{E}_{p}\left[\frac{1}{\sqrt{s}}\right]}{\alpha+\mathbb{E}_{p}\left[\frac{1}{s}\right]}\right)^{2}$. The derivative of $V_{P}^{A}(\alpha, q)$ with respect to $\alpha$ :

$$
\frac{d V_{P}^{A}(\alpha, q)}{d \alpha}=2 \mathbb{E}_{q}^{2}\left[\frac{1}{\sqrt{s}}\right] \frac{d}{d \alpha}\left[\frac{\alpha}{\left(\alpha+\mathbb{E}_{q}\left[\frac{1}{s}\right]\right)^{2}}\right]=\frac{2 \mathbb{E}_{q}^{2}\left[\frac{1}{\sqrt{s}}\right]\left[\mathbb{E}_{q}\left[\frac{1}{s}\right]-\alpha\right]}{\left(\alpha+\mathbb{E}_{q}\left[\frac{1}{s}\right]\right)^{3}}
$$

Setting the derivative to zero, we get a local optimum of the unconstrained problem at $\alpha=\mathbb{E}_{q}\left[\frac{1}{s}\right]$. Further, it follows from (A.21) that $V_{P}^{A}(\alpha, q)$ is increasing for $\alpha<\mathbb{E}_{q}\left[\frac{1}{s}\right]$ and decreasing for $\alpha>\mathbb{E}_{q}\left[\frac{1}{s}\right]$, implying that $\mathbb{E}_{q}\left[\frac{1}{s}\right]$ is a global maximum of the unconstrained problem. If $\mathbb{E}_{q}\left[\frac{1}{s}\right]>1$, the argument of the constrained maxima $\alpha^{N D}$ coincides with $\mathbb{E}_{q}\left[\frac{1}{s}\right]$, and if $\mathbb{E}_{q}\left[\frac{1}{s}\right] \leq 1, \alpha^{N D}$ is given by 1 . Finally, the statement of the lemma follows from the fact that $\mathbb{E}_{q}\left[\frac{1}{s}\right] \lesseqgtr 1 \Leftrightarrow x+q\left(\frac{1}{x}-x\right) \lesseqgtr$ $1 \Leftrightarrow q \lesseqgtr \frac{x}{1+x}$.

Proof of Proposition 3. First, note that Proposition 2, Lemma 6, and Corollary 2 together imply that the principal either chooses $\alpha^{*} \in[x, 1]$ and implements full information disclosure, or chooses $\alpha^{*} \in\left[1, \frac{1}{x}\right]$ and implements no information disclosure. If $\alpha^{*}=1$, the principal's payoff is invariant to the information disclosure policy. The following claims, which characterize the principal's optimal choice in various ranges of $q$, together prove the proposition.

Claim 3. Consider $q>\frac{1}{2}$. Then, $\alpha^{*}=\alpha^{N D}>1$.

Proof of Claim 3: If $\alpha^{*}<1$, by Proposition 2, $\operatorname{Cav}\left(\alpha^{*}, q\right)=V_{P}^{F}\left(\alpha^{*}, q\right)$. Since, $q>\frac{1}{2} \Rightarrow q>\frac{x}{1+x}$, by Lemma 7 and Lemma 8 , we have $\alpha^{F D}>1$ and $\alpha^{N D}>1$, respectively. Therefore, $\max _{\alpha<1} \operatorname{Cav}(\alpha, q)<\underset{\alpha>1}{\max } \operatorname{Cav}(\alpha, q)$, which proves that $\alpha^{*} \nless 1$. Further, 
$\alpha^{*} \neq 1$, since $\alpha^{F D}>1$ and $\alpha^{N D}>1$ and $\operatorname{Cav}(\alpha, q)$ is continuous at $\alpha=1$ by the Maximum theorem. Therefore, $\alpha^{*}>1$. It follows from Corollary 2 that $\alpha^{*}=\alpha^{N D}>1$.

Claim 4. Consider $q<\frac{x}{1+x}$. Then, $\alpha^{*}=\alpha^{F D}<1$.

Proof of Claim 4: If $\alpha^{*}>1$, by Corollary 2, $\operatorname{Cav}\left(\alpha^{*}, q\right)=V_{P}^{A}\left(\alpha^{*}, q\right)$. Further, $q<\frac{x}{1+x} \Rightarrow q<\frac{1}{2}$, and therefore, by Lemmas 7 and 8, we have $\alpha^{F D}<1$ and $\alpha^{N D}=1$, respectively. Therefore, $\max _{\alpha>1} \operatorname{Cav}(\alpha, q)<\underset{\alpha \leq 1}{\max } \operatorname{Cav}(\alpha, q)$, which proves that $\alpha^{*} \ngtr 1$. Further, $\alpha^{*} \neq 1$, since $\alpha^{F D}<1$ and $\alpha^{N D}=1$ and $\operatorname{Cav}(\alpha, q)$ is continuous at $\alpha=1$ by the Maximum theorem. Therefore, $\alpha^{*}<1$. It follows from Proposition 2 that $\alpha^{*}=\alpha^{F D}<1$.

Claim 5. For $\frac{x}{1+x} \leq q \leq \frac{1}{2}$, there exists $\bar{q}$ such that $\alpha^{*}= \begin{cases}\alpha^{F D}<1 & \text { if } q<\bar{q} \\ \alpha^{N D}>1 & \text { if } q>\bar{q} \text {. Further, the principal is indifferent between choos- }\end{cases}$ ing $\alpha=\alpha^{F D}$ and choosing $\alpha=\alpha^{N D}$ if $q=\bar{q}$.

Proof of Claim 5: If $\frac{x}{1+x} \leq q \leq \frac{1}{2}$, by Lemmas 7 and 8, we have $\alpha^{F D} \leq 1 \leq \alpha^{N D}$.

Since $V_{P}^{F}\left(\alpha^{F D}, q\right)=\max _{\alpha>0} V_{P}^{F}(\alpha, q)$, we apply the envelope theorem to get

$$
\begin{aligned}
\frac{d V_{P}^{F}\left(\alpha^{F D}, q\right)}{d q} & =\left.\frac{\partial V_{P}^{F}(\alpha, q)}{\partial q}\right|_{\alpha=\alpha^{F D}}=\left.\frac{\partial}{\partial q} \mathbb{E}_{q}\left[\frac{2 \alpha s}{(1+\alpha s)^{2}}\right]\right|_{\alpha=\alpha^{F D}} \\
& =\frac{2 \alpha^{F D} x}{\left(1+\alpha^{F D} x\right)^{2}}-\frac{2 \alpha^{F D} x}{\left(x+\alpha^{F D}\right)^{2}} \\
& =\frac{2 \alpha^{F D} x\left(1-x^{2}\right)\left(\alpha^{F D}+1\right)\left(\alpha^{F D}-1\right)}{\left(1+\alpha^{F D} x\right)^{2}\left(x+\alpha^{F D}\right)^{2}}
\end{aligned}
$$

which is negative since $\alpha^{F D} \leq 1$.

Similarly, $V_{P}^{A}\left(\alpha^{N D}, q\right)=\max _{\alpha \geq 1} V_{P}^{A}(\alpha, q)$, and applying the envelope theorem, we get

$$
\begin{aligned}
\frac{d V_{P}^{A}\left(\alpha^{N D}, q\right)}{d q} & =\left.\frac{\partial V_{P}^{A}(\alpha, q)}{\partial q}\right|_{\alpha=\alpha^{N D}} \\
& =\frac{4 \alpha^{N D}\left(\alpha^{N D}-1\right)\left(\frac{1}{\sqrt{x}}-\sqrt{x}\right) \mathbb{E}_{q}\left[\frac{1}{\sqrt{s}}\right]}{\left(\alpha^{N D}+\mathbb{E}_{q}\left[\frac{1}{s}\right]\right)^{3}} \text { by (A.11) } \\
& =\frac{\mathbb{E}_{q}\left[\frac{1}{\sqrt{s}}\right]\left(\frac{1}{\sqrt{x}}-\sqrt{x}\right)\left(\alpha^{N D}-1\right)}{2 \mathbb{E}_{q}^{2}\left[\frac{1}{s}\right]},
\end{aligned}
$$

which is positive since $1 \leq \alpha^{N D}$.

The above two observations together imply that $\frac{d}{d q}\left[V_{P}^{A}\left(\alpha^{N D}, q\right)-V_{P}^{F}\left(\alpha^{F D}, q\right)\right]$ is positive and therefore $V_{P}^{A}\left(\alpha^{N D}, q\right)-$ $V_{P}^{F}\left(\alpha^{F D}, q\right)$ is increasing in $q$. Since $V_{P}^{F}(\alpha, q)$ and $V_{P}^{A}(\alpha, q)$ are continuous in $q$, by the Maximum theorem, $V_{P}^{A}\left(\alpha^{N D}, q\right)-$ $V_{P}^{F}\left(\alpha^{F D}, q\right)$ is also continuous in $q$.

Further, $V_{P}^{A}\left(\alpha^{N D}, q\right)-V_{P}^{F}\left(\alpha^{F D}, q\right)>0$ at $q>\frac{1}{2}$ and $V_{P}^{A}\left(\alpha^{N D}, q\right)-V_{P}^{F}\left(\alpha^{F D}, q\right)<0$ at $q<\frac{x}{1+x}$ by Claims 3 and 4 , respectively. Hence, there must exist a threshold $\bar{q} \in\left[\frac{x}{1+x}, \frac{1}{2}\right]$ such that $V_{P}^{A}\left(\alpha^{N D}, q\right)-V_{P}^{F}\left(\alpha^{F D}, q\right) \lesseqgtr 0$ if and only if $q \lesseqgtr \bar{q}$, which proves Claim 5.

Claims 3, 4, and 5 together prove the proposition.

\section{Supplementary material}

Supplementary material associated with this article can be found, in the online version, at doi:10.1016/j.jebo.2021.01.034.

\section{References}

Adamczyk, S., Bullinger, A.C., Möslein, K.M., 2012. Innovation contests: a review, classification and outlook. Creativ. Innov. Manag. 21 (4), 335-360. Azmat, G., Möller, M., 2009. Competition among contests. Rand. J. Econ. 40 (4), 743-768.

Baye, M.R., Kovenock, D., De Vries, C.G., 1993. Rigging the lobbying process: an application of the all-pay auction. Am. Econ. Rev. 83 (1), 289-294.

Chan, W., 1996. External recruitment versus internal promotion. J. Labor. Econ. 14 (4), 555-570.

Che, Y.-K., Gale, I., 1997. Rent dissipation when rent seekers are budget constrained. Public Choice 92 (1-2), 109-126.

Chowdhury, S. M., Esteve-González, P., Mukherjee, A., 2020. Heterogeneity, leveling the playing field, and affirmative action in contests. Available at SSRN: https://ssrn.com/abstract=3655727.

Clark, D.J., Riis, C., 1998. Contest success functions: an extension. Econ. Theory 11 (1), 201-204. 
Denter, P., Morgan, J., Sisak, D., 2020. Showing off or laying low? The economics of psych-outs. Am. Econ. J.: Microecon. forthcoming. Epstein, G.S., Mealem, Y., 2013. Who gains from information asymmetry? Theory Decis. 75 (3), 305-337.

Epstein, G.S., Mealem, Y., Nitzan, S., 2011. Political culture and discrimination in contests. J. Public Econ. 95 (1-2), 88-93.

Epstein, G.S., Mealem, Y., Nitzan, S., 2013. Lotteries vs. all-pay auctions in fair and biased contests. Econ. Polit. 25 (1), $48-60$.

Farrell, S., Hakstian, A.R., 2001. Improving salesforce performance: a meta-analytic investigation of the effectiveness and utility of personnel selection procedures and training interventions. Psychol. Market. 18 (3), 281-316.

Feng, X., Lu, J., 2016. The optimal disclosure policy in contests with stochastic entry: a Bayesian persuasion perspective. Econ. Lett. $147,103-107$.

Franke, J., 2012. Affirmative action in contest games. Eur. J. Polit. Econ. 28 (1), 105-118.

Franke, J., Kanzow, C., Leininger, W., Schwartz, A., 2013. Effort maximization in asymmetric contest games with heterogeneous contestants. Econ. Theory 52 (2), 589-630.

Fu, Q., Lu, J., Zhang, J., 2016. Disclosure policy in Tullock contests with asymmetric stochastic entry. Can. J. Econ./Revue canadienne d'économique 49 (1), $52-75$.

Fu, Q., Wu, Z., 2020. On the optimal design of the biased contest. Theor. Econ. 15 (4), 1435-1470.

Hillman, A.L., Riley, J.G., 1989. Politically contestable rents and transfers. Econom. Polit. 1 (1), 17-39.

Höber, B., 2017. Firm Internal Innovation Contests: Work Environment Perceptions and Employees' Participation. Springer.

Hurley, T.M., Shogren, J.F., 1998a. Asymmetric information contests. Eur. J. Polit. Econ. 14 (4), 645-665.

Hurley, T.M., Shogren, J.F., 1998b. Effort levels in a Cournot Nash contest with asymmetric information. J. Public Econ. 69 (2), 195-210.

Kamenica, E., 2019. Bayesian persuasion and information design. Annu. Rev. Econom. 11, 249-272.

Kamenica, E., Gentzkow, M., 2011. Bayesian persuasion. Am. Econ. Rev. 101 (6), 2590-2615.

Konrad, K.A., Kovenock, D., 2009. Multi-battle contests. Games Econ. Behav. 66, 256-274.

Konrad, K.A., et al., 2009. Strategy and Dynamics in Contests. Oxford University Press, Oxford, UK.

Krishnamoorthy, A., Misra, S., Prasad, A., 2005. Scheduling sales force training: theory and evidence. Int. J. Res. Market. 22 (4), 427-440.

Lu, J., Ma, H., Wang, Z., 2018. Ranking disclosure policies in all-pay auctions with incomplete information. Econ. Inq. 56 (3), $1464-1485$.

Mealem, Y., Nitzan, S., 2016. Discrimination in contests: a survey. Rev. Econ. Des. 20 (2), 145-172.

Rathi, A., 2014. To encourage innovation, make it a competition. Harv. Bus. Rev. 11.

Runkel, M., 2006. Optimal contest design, closeness and the contest success function. Public Choice 129 (1-2), 217-231.

Schaller, Z., Skaperdas, S., 2020. Bargaining and conflict with up-front investments: how power asymmetries matter. J. Econ. Behav. Organ. 176, 212-225.

Serena, M., 2017a. Harnessing beliefs to stimulate efforts. Working Paper of the Max Planck Institute for Tax Law and Public Finance.

Serena, M., 2017b. Quality contests. Eur. J. Polit. Econ. 46, 15-25.

Skiera, B., Albers, S., 1998. Costa: contribution optimizing sales territory alignment. Market. Sci. 17 (3), $196-213$.

Tullock, G., 1980. Efficient Rent Seeking. In: Buchanan, J.M., Tollison, R.D., Tullock, G. (Eds.), Toward a Theory of the Rent-Seeking society. Texas A \& M University Press, College Station, pp. 97-112.

Wärneryd, K., 2003. Information in conflicts. J. Econ. Theory 110 (1), 121-136.

Zhang, J., Zhou, J., 2016. Information disclosure in contests: a Bayesian persuasion approach. Econ. J. 126 (597), $2197-2217$. 